To be published in

\author{
A. V. Narlikar (Ed.) \\ "Frontiers in Superconducting Materials" \\ Springer Verlag, Berlin, 2005
}

\title{
SUPERCONDUCTIVITY OF MAGNESIUM DIBORIDE: THEORETICAL ASPECTS
}

\author{
Thomas Dahm \\ Institut für Theoretische Physik, Universität Tübingen, Auf der Morgenstelle 14, \\ D-72076 Tübingen, Germany
}

\section{INTRODUCTION}

Our theoretical understanding of superconductivity in magnesium diboride $\left(\mathrm{MgB}_{2}\right)$ has made rapid progress since its discovery by Nagamatsu et al. [1]. Unlike superconductivity in the high- $T_{c}$ cuprates we are in a possession of a very clear picture of its superconducting state now [2]. It seems clear that superconductivity is driven by electron-phonon interaction in $\mathrm{MgB}_{2}$. More excitingly, it appears well established both theoretically and experimentally that the rare form of two gap superconductivity is realized in this compound. Two superconducting gaps of distinctively different size appear to exist on different disconnected parts of its Fermi surface. Since $\mathrm{MgB}_{2}$ is the clearest example of two gap superconductivity to date, it makes it an interesting object for study and exploration. In the present work we want to review our present understanding of two gap superconductivity in $\mathrm{MgB}_{2}$ from the theoretical perspective and discuss some of its consequences. The presence of these two different gaps gives rise to a number of anomalies and some of them shall be discussed here. Since the literature on superconductivity in $\mathrm{MgB}_{2}$ has grown rapidly, no complete coverage of experimental and theoretical work can be made here. Instead, the theoretical results most crucial to our understanding in the authors view will be discussed and a selection of peculiar consequences will be presented.

Since much of our present understanding stems from band structure calculations and solutions of Migdal-Eliashberg equations, we are reviewing these results in the next section. The emergence and stability of two gap superconductivity in $\mathrm{MgB}_{2}$ shall be discussed in section 3 . In section 4 we will discuss consequences of this band structure picture for the upper critical field. Section 5 is finally devoted to the microwave conductivity. 


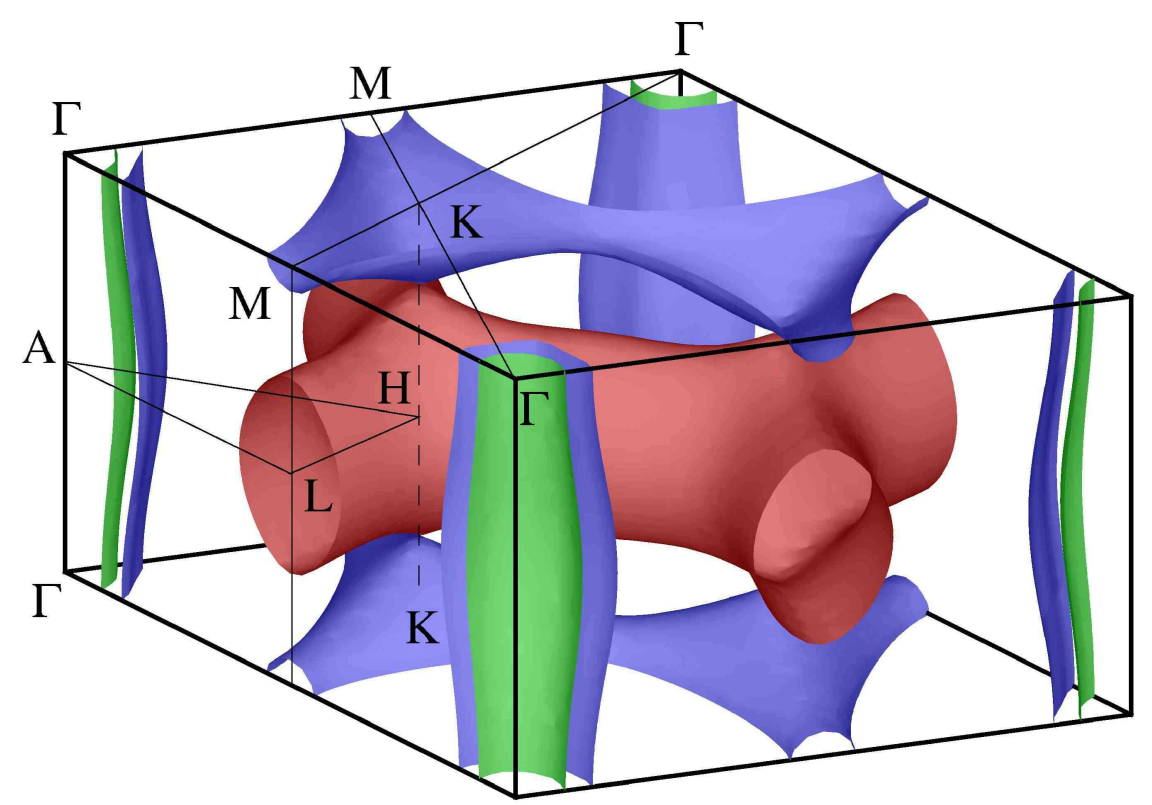

Figure 1: The Fermi surface of $\mathrm{MgB}_{2}$. (Adapted from Ref. [6], (C)2001 The American Physical Society).

\section{THE PICTURE SUGGESTED BY BAND STRUCTURE CALCULATIONS}

Magnesium diboride possesses a comparatively high critical transition temperature compared with other conventional superconductors of about $T_{c}=40 \mathrm{~K}$. Presently only the high- $T_{c}$ cuprates have higher transition temperatures. For that reason the natural question arises whether superconductivity in $\mathrm{MgB}_{2}$ is of the conventional electron-phonon driven type or if its superconducting state has more similarities with the one in the high- $T_{c}$ cuprates. In the high- $T_{c}$ cuprates the superconducting state is of an unconventional $d$-wave type, possessing gap nodes, and electronic pairing mechanisms, like for example exchange of antiferromagnetic spin fluctuations, are discussed seriously. An ongoing discussion still concerns the role and importance of phonons in these compounds.

In contrast to optimally doped high- $T_{c}$ cuprates $\mathrm{MgB}_{2}$ shows a strong isotope effect. If the boron-11 isotope is replaced by the lighter boron-10 isotope, $T_{c}$ increases by about one Kelvin, indicating an important phonon contribution to the pairing interaction [3]. Low temperature specific heat and penetration depth studies are consistent with an exponential decay, indicating the presence of a full gap without nodes [4, 5]. In addition, there are no indications of sizeable magnetic interactions in $\mathrm{MgB}_{2}$, again in contrast to the high- $T_{c}$ cuprates. All these observations seem to place $\mathrm{MgB}_{2}$ among the conventional $s$-wave electron-phonon driven superconductors. Then the question arises, why it has such a high transition temperature as compared with other conventional superconductors. Here, band structure calculations turned out to be elusive, which we want to review in the following.

The $\mathrm{MgB}_{2}$ lattice structure consists of alternating layers of Boron and Magnesium atoms. The Boron atoms form a honeycomb lattice and the Magnesium atoms a triangular lattice halfway between the Boron layers. Calculations of the electronic band structure show four 
bands crossing the Fermi energy leading to four topologically disconnected Fermi surface sheets shown in Fig. 10 [6]. Two of these bands are derived from Boron $\mathrm{p}_{z}$ orbitals. They form the socalled $\pi$ bands seen as the red (electronlike) and blue (holelike) tubular networks in Fig. 11 The other two bands derive from Boron $\mathrm{p}_{x}$ and $\mathrm{p}_{y}$ orbitals and form the so-called $\sigma$ bands, seen as the green and blue cylindrical Fermi surfaces centered around the $\Gamma$ point (both holelike). These possess mainly two dimensional character. Interestingly, all these bands are dominated by Boron $\mathrm{p}$ orbitals and contributions from Magnesium orbitals are very small at the Fermi level. About 58 percent of the total density of states at the Fermi level is residing on the $\pi$ bands making both $\sigma$ and $\pi$ bands about equally important for the electronic properties of $\mathrm{MgB}_{2}$.

Density-functional calculations of the phonon modes and the electron-phonon interaction strength can be found in Refs. [7], [8], and [9]. The highest phonon density of states is found in the energy range around $30 \mathrm{meV}$. However, these phonons only couple weakly to the electrons at the Fermi level and thus do not contribute very much to superconductivity. This can be nicely recognized in Fig. 1 of Ref. [7], where the interaction strength of the phonons is shown as the area of the black circles in the figure. In fact, the phonons that couple most strongly to the electrons at the Fermi level are found in the energy range around $70 \mathrm{meV}$. These phonon modes evolve from the $\mathrm{E}_{2 g}$ mode at the $\Gamma$ point and correspond to a Boron-Boron bond-stretching vibration of the Boron sub lattice. A comparison with the phonon modes in the isostructural but nonsuperconducting compound $\mathrm{AlB}_{2}$ in Ref. [8] shows that these $\mathrm{E}_{2 g}$ phonon modes are strongly softened in $\mathrm{MgB}_{2}$ consistent with their strong coupling. Correspondingly, the so-called Eliashberg function $\alpha^{2} F(\omega)$, which weights the phonon density of states with the coupling strengths and appropriately describes the pairing interaction due to phonons, possesses a strong peak around $70 \mathrm{meV}$ and significantly differs from the phonon density of states in contrast to conventional strong-coupling superconductors. The dimensionless electron-phonon coupling constant was found to lie between $\lambda \approx 0.7-0.9$ from these first-principles calculations. From this microscopic information we can obtain a qualitative understanding of why $T_{c}$ is so high in $\mathrm{MgB}_{2}$ by looking at the BCS $T_{c}$ formula (which is of course a bad approximation in the present case, but can give us some qualitative tendencies):

$$
k_{B} T_{c}=1.13 \hbar \omega_{c} e^{-1 / V N(0)}
$$

Here, $\omega_{c}$ is a characteristic phonon frequency, $V$ the interaction strength and $N(0)$ the density of states at the Fermi level, with $\lambda \sim V N(0)$. First of all, the characteristic phonon frequency is comparatively high, because Boron is a light element and the $\mathrm{E}_{2 g}$ phonon modes in question only involve vibrations of the Boron sub lattice. Secondly, this high frequency phonon at the same time possesses a strong coupling to the electrons at the Fermi level. This means that in $\mathrm{MgB}_{2}$ we have a favorable coincidence of two effects helping to raise $T_{c}$.

\section{TWO GAP SUPERCONDUCTIVITY IN MAGNESIUM DIBORIDE}

It was noted already early on that there is a problem with the superconducting gap size in $\mathrm{MgB}_{2}$. From the BCS gap ratio $\Delta_{0}=1.76 k_{B} T_{c}$ one should expect a gap of $6 \mathrm{meV}$ or somewhat larger, if strong electron-phonon coupling effects are considered. However, experimental results obtained from different experimental techniques seemed to scatter between $2 \mathrm{meV}$ and $8 \mathrm{meV}$ with some clustering around $2.5 \mathrm{meV}$ and $7 \mathrm{meV}$ [10]. Initially, one might have attributed this to insufficient sample quality, however, by the end of 2001 high quality single 
crystals became available and this problem remained. Also, values of the coherence length $\xi$ extracted by different means turned out to differ considerably. For example, STM tunneling measurements of the vortex core size by Eskildsen et al. [11] were consistent with a value of $\xi \approx 50 \mathrm{~nm}$, while the coherence length as determined from the upper critical field value on the same sample gave $\xi \approx 10 \mathrm{~nm}$. Since the coherence length is related to the superconducting gap via $\xi=\hbar v_{F} / \pi \Delta_{0}$ these differences can be related to differences in the gap value as well. These problems suggested studying the possibility of anisotropic or multiple gap structures in $\mathrm{MgB}_{2}$.

Again, important insight into this question was provided by Migdal-Eliashberg type calculations based on band structure calculations [9, 12, 13, 14]. Migdal-Eliashberg theory is a generalization of BCS-theory and allows to take into account the detailed properties of phonons and their coupling to the electrons at the Fermi level in the pairing interaction. Decomposing the electron-phonon coupling constant into contributions from the four bands Liu et al. [9] were able to show that the pairing interaction differs considerably on the $\sigma$ and $\pi$ bands. The pairing interaction on the $\sigma$ bands turns out to be much stronger than the one on the $\pi$ bands and the interband pairing strength. The reason for this is that the $\mathrm{E}_{2 g}$ phonon mode being an in-plane vibration of the Boron atoms preferentially couples to the in-plane electrons on the more two dimensional $\sigma$ bands, while its coupling to the three dimensional $\pi$ bands is much weaker. Due to this anisotropy the resulting effective coupling constant was shown to increase to about $\lambda \approx 1$, being quantitatively more consistent with a $T_{c}$ of $40 \mathrm{~K}$.

A fully momentum dependent solution of Migdal-Eliashberg equations was presented by H. J. Choi et al in Refs. [13] and [14]. In this calculation the fully momentum dependent band structure, phonon modes, and electron-phonon couplings obtained from first principles density functional calculations were used as a starting point to solve Migdal-Eliashberg equations in the full Brillouin zone. The Coulomb pseudopotential was taken as a constant $\mu^{*}=0.12$, which reproduces the experimental $T_{c}$. Neglecting impurity scattering, this procedure allowed to calculate the value of the superconducting gap at each point on the Fermi surface. The result is shown in Fig. 2, where the size of the gap is shown color coded on top of the Fermi surface structure. This picture impressively demonstrates the presence of a large gap value around 7 meV on the $\sigma$ bands and a small gap value around $2 \mathrm{meV}$ on the $\pi$ bands.

Consistent with Ref. [9] the authors were able to demonstrate the important role of both anisotropy and anharmonicity of the phonons on $T_{c}$. When anisotropy was neglected and only the isotropic Migdal-Eliashberg equations were solved, $T_{c}$ dropped from $39 \mathrm{~K}$ to $19 \mathrm{~K}$. When anharmonicity on the phonon frequencies was neglected but anisotropy was kept, $T_{c}$ was seen to increase from $39 \mathrm{~K}$ to $55 \mathrm{~K} \mathrm{[13]}$. This shows that phonon anharmonicity is harmful for superconductivity in $\mathrm{MgB}_{2}$, but anisotropy is helpful, and for a quantitative understanding of superconductivity in $\mathrm{MgB}_{2}$ both are needed. Choi et al. were also able to show that the isotope exponent observed experimentally could be quantitatively reproduced, if the anharmonicity of the phonons was taken into account. Also, the temperature dependence of the specific heat from these calculations was in good agreement with the experimental results. All these results seem to show that we can obtain a complete understanding in terms of two gap superconductivity in $\mathrm{MgB}_{2}$ based on the picture shown in Fig. 2

An important question in this picture concerns the role of impurities and in particular interband impurity scattering, however. Conventionally one would expect that impurity scattering tends to equalize all gap values on the Fermi surfaces thereby reducing $T_{c}$. This is one of the 


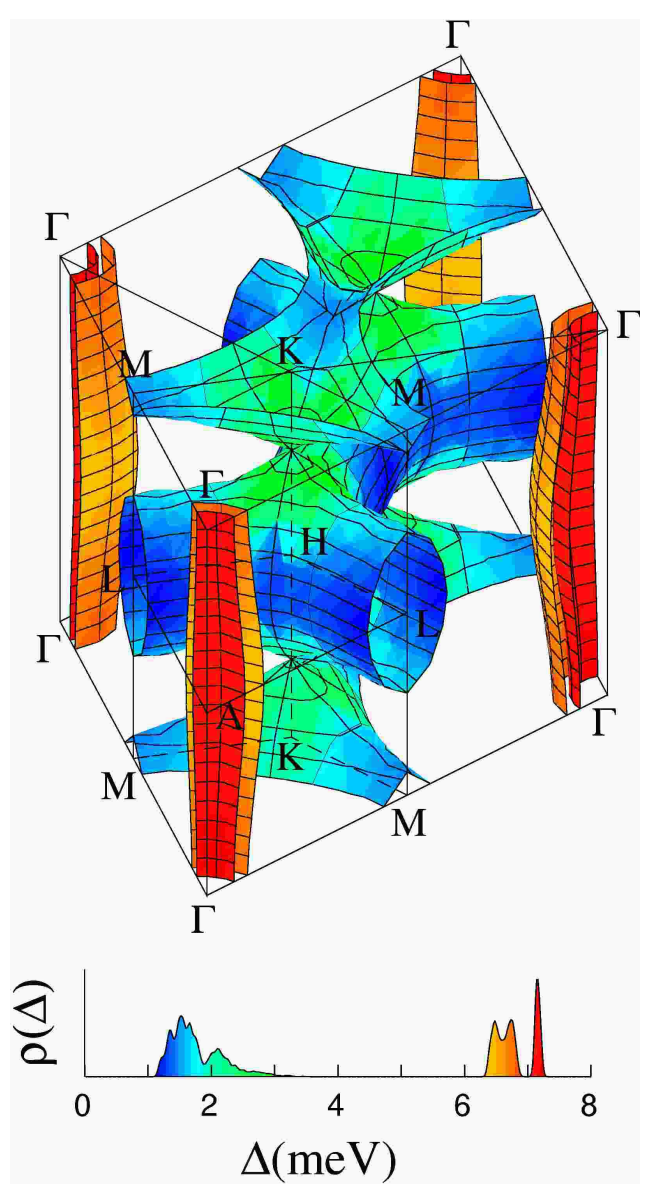

Figure 2: The energy gap distribution on the Fermi surface of $\mathrm{MgB}_{2}$ (color coded). (Adapted from Ref. [14], (c)2002 Nature Publishing Group).

reasons why two gap superconductivity or anisotropic $s$-wave superconductivity is rarely observed. This does not seem to be the case in $\mathrm{MgB}_{2}$. For example, samples with very different residual resistivities are observed to have essentially the same critical temperature $T_{c}$. This is a behavior that one would expect in a single gap $s$-wave superconductor due to Anderson's theorem. However, in a two gap superconductor a strong change of $T_{c}$ with impurity concentration should be expected [15]. A very good answer to this question has been given by I. I. Mazin et al. in Ref. [16]: due to the particular electronic structure of $\mathrm{MgB}_{2}$ the electronic wave functions on $\pi$ bands and $\sigma$ bands possess different parity symmetry. The $\pi$ bands deriving from the Boron $\mathrm{p}_{z}$ orbitals are mainly antisymmetric with respect to the Boron plane, while the $\sigma$ bands deriving from both Boron $\mathrm{p}_{x}$ and $\mathrm{p}_{y}$ orbitals are mainly symmetric. This disparity of $\sigma$ and $\pi$ bands makes the impurity scattering matrix element between these two types of bands exceptionally small as compared with the impurity scattering matrix element within each of these bands. Using density functional supercell calculations for various impurities Mazin et al were able to show that the interband scattering rate appears to be one to two orders of magnitude smaller than the intraband scattering rates due to this band disparity. This means that 
impurity scattering will equalize the gaps within each of the two types of bands, but not very much between them. This makes the two gaps in $\mathrm{MgB}_{2}$ exceptionally stable against impurity scattering. Only a very large amount of impurity scattering is expected to lead to a reduction of $T_{c}$ due to interband scattering and an accompanied merging of the two gaps.

While signatures of the two gaps have been observed in several different experiments, so far direct experimental confirmation of the important role of the $\mathrm{E}_{2 g}$ phonon for the pairing is still lacking. In principle, such a strong coupling phonon could produce observable structures in the tunneling density of states. However, there are difficulties in the case of $\mathrm{MgB}_{2}$ as has been discussed by Dolgov et al [17]: on one hand the high frequency of the phonon mode leads to a strong reduction of the structures produced in the tunneling spectrum, on the other hand tunneling spectroscopy is mostly dominated by the $\pi$ band phonons, which are not so important for the pairing interaction. This makes observation of the $\mathrm{E}_{2 g}$ phonon and its coupling strength in tunneling spectroscopy difficult.

Concluding this section we can say that we seem to have a consistent and quantitative picture of two gap superconductivity in $\mathrm{MgB}_{2}$, which mainly arises from density functional calculations and solutions of Migdal-Eliashberg equations. The two gaps arise due to a strongly anisotropic electron-phonon interaction of the (anharmonic) $\mathrm{E}_{2 g}$ phonon mode, which couples more strongly to the electrons on the $\sigma$ bands than to the ones on the $\pi$ bands. Due to the disparity of the two types of bands these two gaps appear to be very stable against impurity scattering, allowing $T_{c}$ to remain large even in samples with large residual resistivities.

\section{UPPER CRITICAL FIELD ANISOTROPY}

In this section we want to explore the consequences of the two gap picture presented in the previous section on the temperature dependence of the upper critical field $B_{c 2}$, particularly its anisotropy. Measurements of the upper critical field in $\mathrm{MgB}_{2}$ single crystals have shown that not only $B_{c 2}$ is anisotropic, but also that this anisotropy is strongly temperature dependent [18, 19, 20, 21]. The anisotropy ratio $\Gamma_{c 2}$ is given by $\Gamma_{c 2}=B_{c 2}^{a b} / B_{c 2}^{c}$, where $B_{c 2}^{a b}$ is the upper critical field, when the field is applied along the Boron planes, and $B_{c 2}^{c}$ the one for field along $c$-axis direction perpendicular to the Boron planes. At low temperatures $\Gamma_{c 2}$ reaches values around 5. It decreases with increasing temperature and reaches values around 2 at $T_{c}$ (see the solid circles in Fig. 4 below). Close to $T_{c} B_{c 2}^{c}$ varies linearly with temperature, while $B_{c 2}^{a b}$ shows a pronounced upward curvature. This behavior is quite unusual, because in conventional single gap superconductors the anisotropy ratio is very much temperature independent, rarely changing by more than 10 to 20 percent. An upward curvature of the upper critical field has been observed in $\mathrm{YNi}_{2} \mathrm{~B}_{2} \mathrm{C}$ and attributed to two band behavior, however, in this case the upward curvature appeared in all spatial directions, in contrast to $\mathrm{MgB}_{2}$ [22].

The first theoretical work addressing this unusual behavior in $\mathrm{MgB}_{2}$ was a study within an anisotropic gap model by Posazhennikova et al. [23]. In this work a single anisotropic $s$-wave gap on an elliptical Fermi surface was considered. It was shown that the experimental data of the upper critical field in $\mathrm{MgB}_{2}$ single crystals including both the strong temperature dependence of the anisotropy ratio as well as the upward curvature only appearing with field in $a b$-plane direction could be fitted within this model, if the gap was smaller in $c$-axis direction than in $a b$ plane direction. While this model can account for all experimental observations on the upper critical field in $\mathrm{MgB}_{2}$, it possesses some drawbacks, however, regarding its consistency with 
other experimental results. One problem concerns the ratio of the maximum to the minimum gap value. Within this anisotropic $s$-wave model a gap ratio of about 1:10 was needed to explain the upper critical field data. This ratio seems too large compared with the about 1:3 ratio as observed in tunneling experiments, for example. In addition, measurements of the in-plane penetration depth clearly show an exponential decrease with the small gap [24, 25]. This suggests that the small gap is present within the Boron plane direction consistent with the picture in Fig. 2, but inconsistent with this anisotropic $s$-wave model.

Another problem concerns the anisotropy of the lower critical field $B_{c 1}$. From anisotropic (single gap) Ginzburg-Landau theory one should expect that the upper critical field anisotropy is related to the lower critical field anisotropy via $B_{c 2}^{a b} / B_{c 2}^{c}=B_{c 1}^{c} / B_{c 1}^{a b}$. Recent experimental studies have established, however, that this relation is violated in $\mathrm{MgB}_{2}$ [26, 27, 28], the upper critical field anisotropy decreasing with increasing temperature, while the lower critical anisotropy is found to increase. This is in agreement with expectations based on two gap models [29], but again inconsistent with an anisotropic $s$-wave model.

A first calculation within an anisotropic two band model was presented by Miranovic et al. [29] based on clean limit Eilenberger theory. The Fermi surface sheets of the two bands were taken as ellipsoids with very different anisotropies, while the two gaps were taken to be isotropic. Within this calculation it was also possible to qualitatively reproduce the experimentally observed upper critical field anisotropy as in the anisotropic $s$-wave model by Posazhennikova et al. In addition to that, however, this two band calculation did not suffer from the drawbacks mentioned above: more realistic values for the ratio of the two gaps could be taken, the small gap was present within the Boron plane direction, and the lower critical field anisotropy was found to increase with temperature.

In order to answer the question whether the picture in Fig. 2] is consistent with the upper critical field anisotropy the author and N. Schopohl presented a calculation within two band Eilenberger theory taking into account the detailed band structure Fermi surface topology based on Fig. 1 [30]. For that purpose a simple but realistic model of the Fermi surface was used as shown in Fig. 3 The upper panel of Fig. 3 shows one half of the Brillouin zone from Fig. 1 Using the periodicity of the lattice identical copies of the Brillouin zone may be attached to each other (center panel). Choosing a new Brillouin zone around the $\Gamma$ point leads to the structure shown in the lower panel. This rearrangement shows that the $\sigma$ band Fermi surface can be modeled by a distorted cylinder and the $\pi$ band Fermi surface by a torus cut open half. Here, the two $\sigma$ bands and the two $\pi$ bands are assumed to be degenerate, for simplicity. This suggests the following parameterization of the Fermi surfaces for the $\sigma$ band Fermi momentum [31]:

$$
\vec{k}_{F, \sigma}\left(\varphi, k_{z}\right)=\left(\begin{array}{c}
\left(k_{F, \sigma}+\frac{\epsilon_{c}}{c} \cos \left(c k_{z}\right)\right) \cos \varphi \\
\left(k_{F, \sigma}+\frac{\epsilon_{c}}{c} \cos \left(c k_{z}\right)\right) \sin \varphi \\
k_{z}
\end{array}\right)
$$

and the $\pi$ band Fermi momentum:

$$
\vec{k}_{F, \pi}(\varphi, \vartheta)=\left(\begin{array}{c}
k_{F, \pi}\left(\frac{1}{\kappa}+\cos \vartheta\right) \cos \varphi \\
k_{F, \pi}\left(\frac{1}{\kappa}+\cos \vartheta\right) \sin \varphi \\
k_{F, \pi} \sin \vartheta
\end{array}\right)
$$



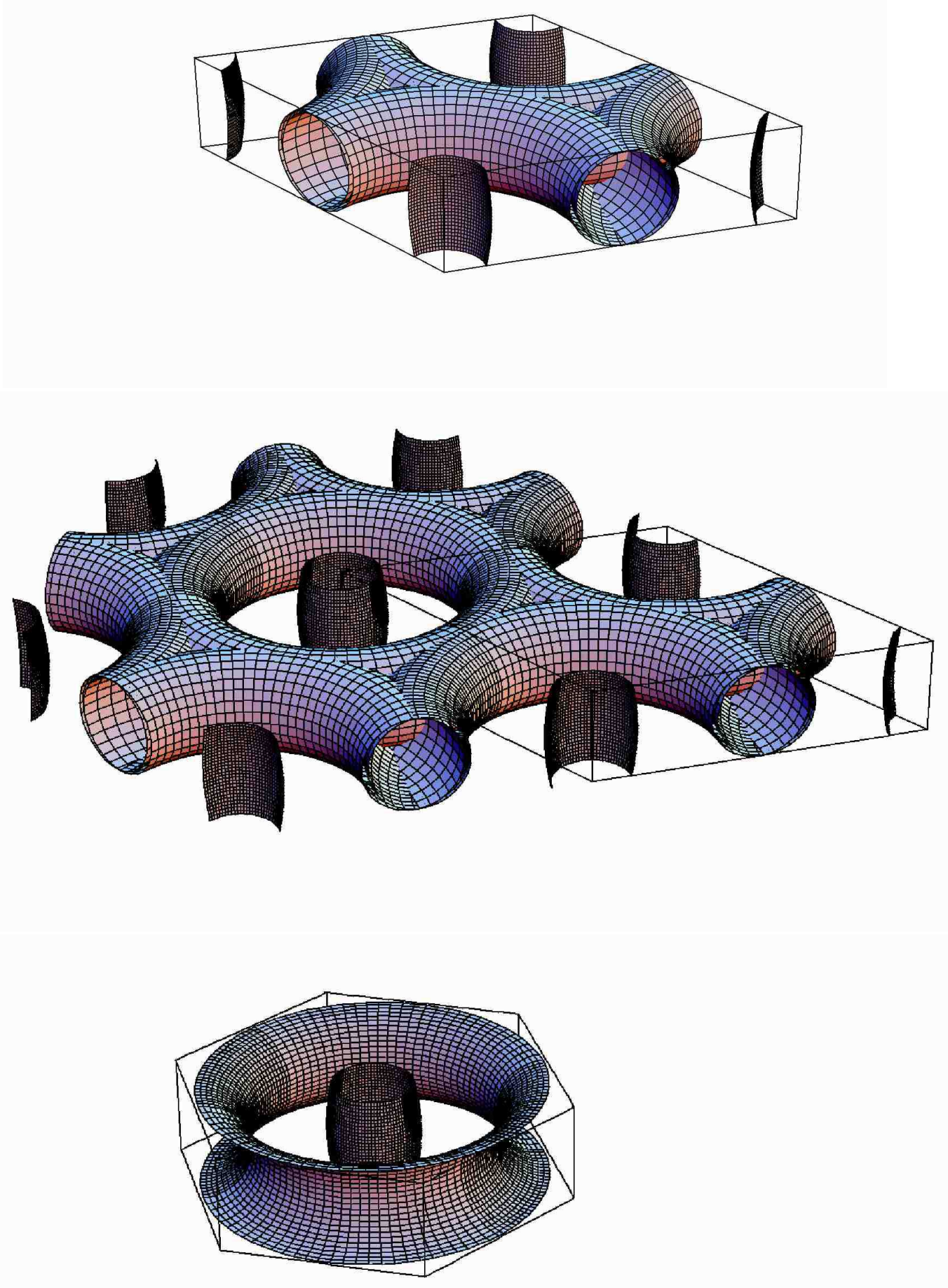

Figure 3: Simplification of the $\mathrm{MgB}_{2}$ Fermi surface from Fig. 1. Rearranging the Fermi surface sheets around the $\Gamma$ point shows that the $\sigma$ band can be modeled as a distorted cylinder and the $\pi$ band as a half-torus. 
Here, $\varphi$ is the in-plane angle, $k_{z}$ the $c$-axis momentum, and $\vartheta$ the azimuthal angle of the halftorus running from $\pi / 2$ to $3 \pi / 2$. The corresponding Fermi velocities are given by

$$
\vec{v}_{F, \sigma}\left(\varphi, k_{z}\right)=v_{F, \sigma}\left(\begin{array}{c}
\cos \varphi \\
\sin \varphi \\
\epsilon_{c} \sin c k_{z}
\end{array}\right) \quad \text { and } \quad \vec{v}_{F, \pi}(\varphi, \vartheta)=v_{F, \pi}\left(\begin{array}{c}
\cos \varphi \cos \vartheta \\
\sin \varphi \cos \vartheta \\
\sin \vartheta
\end{array}\right)
$$

Here, the dimensionless $c$-axis dispersion parameter $\epsilon_{c}$ has been assumed small. For $\mathrm{MgB}_{2}$ the following parameter values have been extracted from band structure calculations [30]: $v_{F, \sigma}=$ $4.4 \times 10^{5} \mathrm{~m} / \mathrm{s}, v_{F, \pi}=8.2 \times 10^{5} \mathrm{~m} / \mathrm{s}, \epsilon_{c}=0.23$, and $\kappa=0.25$.

Based on this model for the $\mathrm{MgB}_{2}$ Fermi surface one can now calculate the upper critical field using the linearized two band Eilenberger equations. For $\omega_{n}>0$ they read

$$
\left\{\omega_{n}+\vec{v}_{F, \alpha}\left[\frac{\hbar}{2} \vec{\nabla}-i \frac{e}{c} \vec{A}(\vec{r})\right]\right\} f_{\alpha}\left(\vec{r}, \hat{k} ; \omega_{n}\right)=-\Delta_{\alpha}(\vec{r})
$$

along with the gap equation

$$
\Delta_{\alpha}(\vec{r})=-\pi T \sum_{\alpha^{\prime}} \sum_{\left|\omega_{n}^{\prime}\right|<\omega_{c}} \lambda^{\alpha \alpha^{\prime}}\left\langle f_{\alpha^{\prime}}\left(\vec{r}, \hat{k}^{\prime} ; \omega_{n}^{\prime}\right)\right\rangle_{\alpha^{\prime}}
$$

Here, $f_{\alpha}$ is the anomalous Eilenberger propagator and $\Delta_{\alpha}(\vec{r})$ the (spatially dependent) gap function for the two bands $\alpha \in\{\pi, \sigma\}$. The pairing interaction $\lambda^{\alpha \alpha^{\prime}}$ becomes a two-by-two matrix in the band indices. The effective matrix elements have been calculated from band structure calculations in Ref. [9] and found to be

$$
\left(\begin{array}{ll}
\lambda^{\sigma \sigma} & \lambda^{\sigma \pi} \\
\lambda^{\pi \sigma} & \lambda^{\pi \pi}
\end{array}\right)=\left(\begin{array}{ll}
0.959 & 0.222 \\
0.163 & 0.278
\end{array}\right)
$$

Together Equations (5) and (6) establish an eigenvalue problem for the gap function $\Delta_{\alpha}(\vec{r})$. For a given temperature $T$ one has too look for the solution $\Delta_{\alpha}(\vec{r})$ that solves both equations for the highest value of the magnetic field $\vec{B}=\vec{\nabla} \times \vec{A}$.

Eilenberger theory is a generalization of BCS theory to inhomogeneous superconducting states and contains Ginzburg-Landau theory as a limiting case for $T \rightarrow T_{c}$ [36, 37]. It holds in the limit $k_{F} \xi \ll 1$, where $\xi$ is the coherence length and $k_{F}$ the Fermi momentum. Since Ginzburg-Landau theory is limited to the vicinity of $T_{c}$, Eilenberger theory is the method of choice, if one wants to calculate properties of type-II superconductors in the vortex state at lower temperatures from microscopic grounds. Near the upper critical field the gap function becomes small and therefore one can use the linearized Eilenberger equations to determine $B_{c 2}$.

Equations (5) and (6) are usually solved using a Landau level expansion of $\Delta_{\alpha}(\vec{r})$ above the Abrikosov ground state of the vortex lattice [32]. For strongly anisotropic systems this procedure requires a high number of excited states, however. In addition, the calculation can only be done numerically. In Ref. [30] Equations (5) and (6) were solved using a variational method that had been introduced earlier by the author in Ref. [23]. This method does not require a high number of excited states and even allows to obtain analytical results in some limiting cases. The variational ansatz for $\Delta_{\alpha}(\vec{r})$ corresponds to a distorted Abrikosov ground state of the form

$$
\Delta_{\alpha}(\vec{r})=\Delta_{\alpha} \psi_{\Lambda}\left(e^{-\tau} x, e^{\tau} y\right)
$$




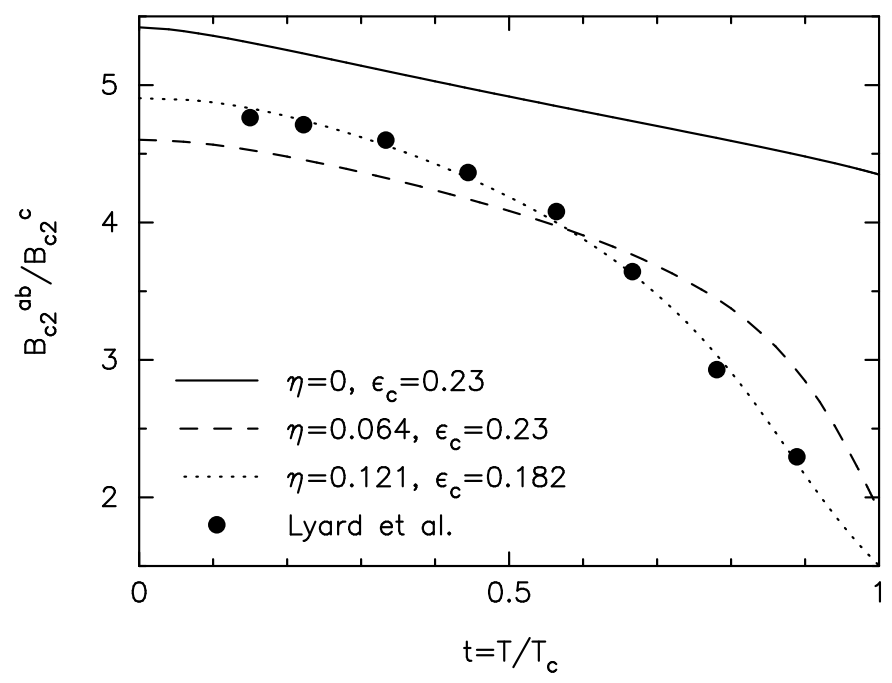

Figure 4: Temperature dependence of the anisotropy ratio $\Gamma_{c 2}=B_{c 2}^{a b} / B_{c 2}^{c}$ for the band structure based two-band model of Ref. [30]. Results are shown for different interband pairing strengths $\eta$ and $c$-axis dispersions $\epsilon_{c}$. Solid circles are experimental results from Lyard et al. [20] (adapted from Ref. [30], (c)2003 The American Physical Society).

where $\tau$ is used as a variational parameter and determined such as to maximize the upper critical field $B_{c 2}$. Here, $\psi_{\Lambda}$ is the usual (undistorted) Abrikosov ground state. More details about this variational method can be found in the appendix.

Using this method the temperature dependence of the anisotropy ratio was calculated from microscopic grounds based on band structure calculations in Ref. [30] as pointed out above. The result is shown in Fig. 4. Here, the parameter $\eta$ is a dimensionless parameter describing the interband pairing strength and is given by

$$
\eta=\frac{\lambda^{\pi \pi}-\lambda_{-}}{\lambda_{+}-\lambda_{-}}
$$

where $\lambda_{+}$and $\lambda_{-}$are the larger and smaller eigenvalues of the matrix $\lambda^{\alpha \alpha^{\prime}}$ in Eq. (6), respectively. The dashed line shows the result for the parameters obtained from band structure calculations, the solid circles are experimental results on $\mathrm{MgB}_{2}$ single crystals from Lyard et al. [20]. For comparison, also the result with no interband pairing $\eta=0$ is shown as the solid line. In this case the upper critical field anisotropy is determined by the $\sigma$ band only.

In Ref. [30] it was found that the two most important parameters determining the temperature dependence of the upper critical field anisotropy are $\eta$ and the $c$-axis dispersion $\epsilon_{c}$ of the cylindrical $\sigma$ band. All other parameters have only minor influence on the shape of the curves in Fig. (4 If these two parameters are allowed to vary from their band structure values, one can obtain an excellent fit of the experimental data shown as the dotted line in Fig. 4. In this (two parameter) fit the interband pairing strength $\eta$ turns out to be somewhat larger and the $c$-axis dispersion $\epsilon_{c}$ a little bit smaller than expected from band structure calculations. Similar observations, that larger interband pairing strengths than found by band structure calculations are needed to fit experimental data, have been made also in Refs. [33] and [34]. Possible 


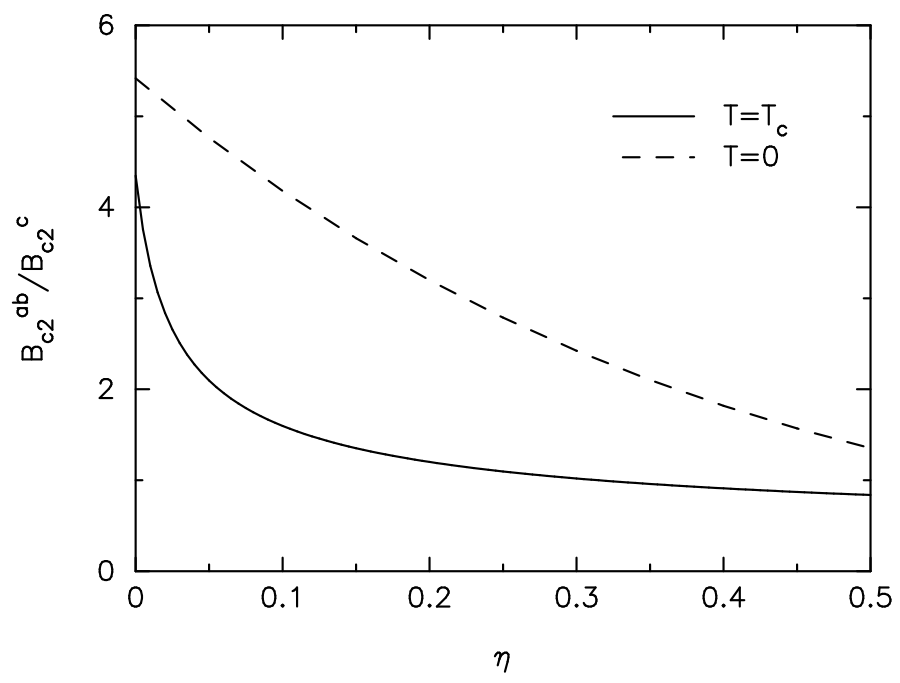

Figure 5: Anisotropy ratio $\Gamma_{c 2}=B_{c 2}^{a b} / B_{c 2}^{c}$ as a function of interband pairing strength $\eta$ for the band structure based two-band model of Ref. [30] at $T=0$ (dashed line) and $T=T_{c}$ (solid line) (adapted from Ref. [30], (c)2003 The American Physical Society).

explanations could be either that band structure calculations underestimate the interband pairing strength or that some small amount of interband impurity scattering or effects of strong electron-phonon coupling, which were neglected in the above calculations of the upper critical field, mimic a somewhat stronger interband pairing strength.

The result in Fig. 4 shows that indeed a strong temperature dependence of the upper critical field ratio consistent with experimental results has to be expected in the clean limit for the parameters found in band structure calculations for $\mathrm{MgB}_{2}$. As the solid line shows, the influence of both gaps is crucial here. The strong temperature dependence can be understood as follows: at low temperatures and high magnetic fields the $\sigma$ band with the large gap is dominating leading to a strong anisotropy. When temperature is increased and thus the magnetic field reduced, the influence of the $\pi$ band with the small gap becomes more and more important. Since the $\pi$ band is more isotropic a reduction of the upper critical field anisotropy results.

It is instructive to look at the change of $\Gamma_{c 2}$ as a function of the interband pairing strength $\eta$ at $T=0$ and $T=T_{c}$. This is shown in Fig. 5. When $\eta=0$ there is no strong temperature dependence of $\Gamma_{c 2}$. When $\eta$ is increased, $\Gamma_{c 2}$ decreases much more rapidly at $T=T_{c}$ than at $T=0$, because the influence of the $\pi$ band is more important at higher temperature. As a result the temperature dependence becomes stronger. However, when $\eta$ is increased towards 0.5 (maximum coupling of the two bands), eventually $\Gamma_{c 2}$ becomes small also at low temperature and the temperature dependence becomes weak again. It appears that either for very weak or for very strong interband pairing interaction the system effectively behaves like a single gap superconductor with a weak temperature dependence of the upper critical field anisotropy. It is only in the intermediate regime around $\eta \sim 0.05-0.3$ where a strong temperature dependence of $\Gamma_{c 2}$ can be observed. This is just the regime in which $\mathrm{MgB}_{2}$ appears to be according to band structure calculations.

In Ref. [35] further results of this model can be found: the temperature dependence of the 
upper critical field for field applied along the Boron plane direction shows an upward curvature, while for field applied in $c$-axis direction it does not, in good agreement with the experiments. Also, the magnetic field dependence of the zero energy density of states follows the experimental temperature dependence of the electronic specific heat coefficient.

The theories about the upper critical field in $\mathrm{MgB}_{2}$ discussed so far are clean limit theories, e.g. impurity scattering was assumed to be small. However, even in the best $\mathrm{MgB}_{2}$ single crystals available so far it is believed that in the $\pi$ band the scattering rate is larger than the gap, while in the $\sigma$ band the scattering rate might be smaller than the gap. This means that impurities are expected to affect the temperature dependence of the upper critical field and one might ask how this could affect the clean limit results discussed above. So far no calculations for general impurity scattering rates have been presented. However, calculations can be considerably simplified in the dirty limit. In Refs. [38, 39] such dirty limit theories of the upper critical field for $\mathrm{MgB}_{2}$ were presented. The main simplification here is that the intraband scattering rates $\Gamma_{\pi}$ and $\Gamma_{\sigma}$ are assumed to be larger than the gaps, while the interband scattering rate is assumed to be negligible due to the parity argument by Mazin et al. [16] discussed in the previous section. Interestingly, the strong temperature dependence of the upper critical field anisotropy was shown to exist also in this dirty limit [39]. The physical reason for this behavior is the same as in the clean limit: an interband pairing interaction of intermediate strength and a strong anisotropy of the Fermi velocities in the $\sigma$ band which results in a strong anisotropy of the diffusivities in this band. Therefore, the inclusion of impurities qualitatively does not change this behavior.

In Ref. [39] also the angular dependence of the upper critical field was studied. It was shown that this angular dependence shows strong deviations from the results expected from anisotropic Ginzburg-Landau theory. The reason for this is that the $c$-axis coherence lengths in the two bands strongly differ. As a result the validity of Ginzburg-Landau theory is reduced to a very narrow region near the critical temperature $T_{c}$ [40].

To summarize this section we have seen that the unusually strong temperature dependence of the upper critical field anisotropy can be nicely understood in terms of the two gap model, if the particular Fermi surface topology of $\mathrm{MgB}_{2}$ is taken into account. The essential ingredients for this effect are two Fermi surfaces with very different anisotropies and an interband pairing interaction with an intermediate strength. Too weak or too strong interband pairing would result in effectively single gap behavior. According to band structure calculations $\mathrm{MgB}_{2}$ fulfils these requirements possessing a strongly anisotropic cylindrical $\sigma$ band and a more isotropic toroidal $\pi$ band. Parameters from band structure calculations can even reproduce the upper critical field anisotropy quantitatively. This shows that the behavior of the upper critical field is consistent with the band structure picture presented in the previous sections.

\section{MICROWAVE CONDUCTIVITY}

In this section we want to discuss the consequences of two gap superconductivity on the microwave conductivity in $\mathrm{MgB}_{2}$, particularly its temperature dependence at fixed frequency. In Ref. [33] the observation of an anomalous microwave conductivity peak in $\mathrm{MgB}_{2}$ thin films has been reported and its interpretation in terms of two gap behavior shall be discussed here. We will first start with a review of conductivity peaks in conventional superconductors as well as in high- $T_{c}$ cuprates and then compare them with the results in $\mathrm{MgB}_{2}$.

In conventional dirty limit superconductors the microwave conductivity at frequencies suf- 


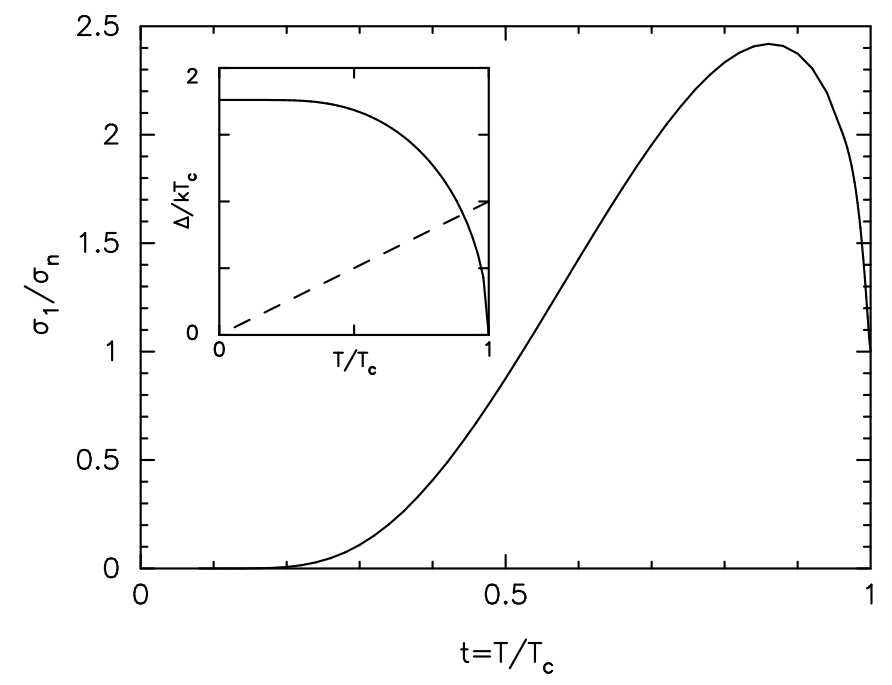

Figure 6: Temperature dependence of the microwave conductivity at a frequency of $\omega=$ $0.02 k_{B} T_{c}$ for a conventional $s$-wave superconductor in the dirty limit. Inset: temperature dependence of the gap (solid line) along with the line $\Delta=k_{B} T$ (dashed line). The crossing point roughly determines the position of the peak in the conductivity.

ficiently below the gap frequency shows a coherence peak as a function of frequency, which is related to the so-called Hebel-Slichter peak in the temperature dependence of the NMR spin relaxation rate. As shown in Fig. 6 the conductivity initially increases when entering the superconducting state going through a peak value near $0.9 T_{c}$ and finally being suppressed exponentially at lower temperatures. The peak naturally comes out of BCS theory and at the time was regarded as one of the key triumphs of BCS theory, because earlier theories based on two fluid models were not able to account for this effect. When the superconducting state is entered and the gap opens, a square-root singularity appears in the density of states at the gap edge. This singularity leads to increased contributions to the microwave conductivity. When the gap $\Delta$ increases upon lowering the temperature, it eventually becomes larger than $k_{B} T$. At this point quasiparticles are condensed out into the superfluid and the microwave conductivity is suppressed exponentially at lower temperatures. As a rule of thumb we can estimate the position of the coherence peak roughly at the point where $\Delta(T)$ becomes equal to $k_{B} T$, which is shown as the crossing point between the solid and the dashed line in the inset of Fig. 6, In contrast to the NMR Hebel-Slichter peak, the coherence peak in the microwave conductivity depends on the scattering rate, however. When the system becomes cleaner, the microwave coherence peak is gradually reduced, as has been discussed by Marsiglio [41]. The ultimate reason for this dependence on the scattering rate is lying in the fact that the conductivity is a nonlocal quantity in contrast to the NMR relaxation rate which is a local probe.

In the high- $T_{c}$ cuprates the situation is completely different. In measurements of the NMR relaxation rate no Hebel-Slichter peak is observed. However, the microwave conductivity shows a huge peak at temperatures between $0.3 T_{c}$ and $0.6 T_{c}$ depending on frequency as shown in Fig. 7. The absence of the Hebel-Slichter peak can be easily understood as a consequence of the $d$-wave nature of the superconducting state. In a $d$-wave superconductor the singularity at 


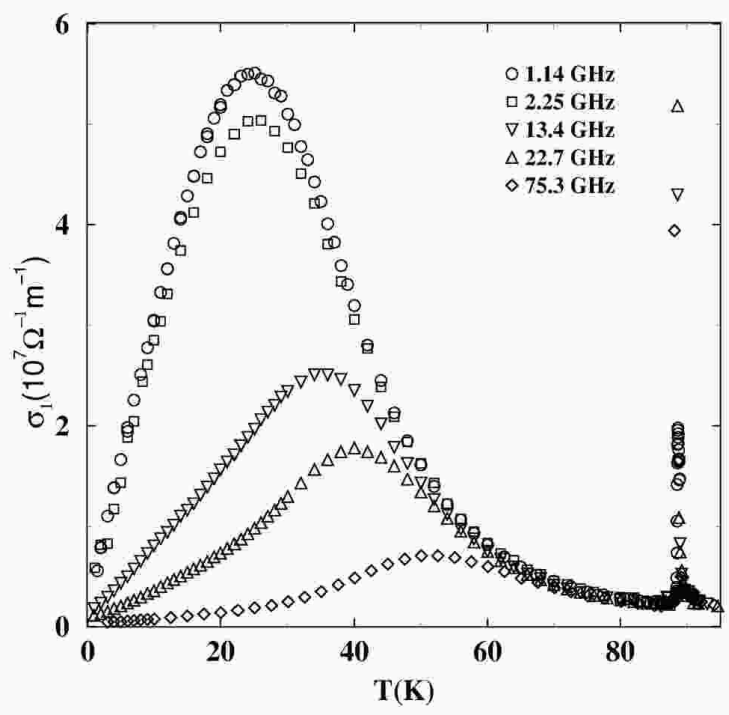

Figure 7: Temperature dependence of the microwave conductivity in YBCO showing a large conductivity peak (adapted from Ref. [42], (C)1999 The American Physical Society).

the gap edge of the density of states is not a square-root singularity anymore, but a logarithmic singularity, having a much weaker influence on the NMR relaxation rate, as shown in Fig. 8 In addition, strong-coupling effects tend to wash out the singularities in the density of states, which leads to an additional suppression of the coherence peak as discussed in Ref. [41].

Since there is no coherence peak in the NMR relaxation rate, why is there a peak in the microwave conductivity? It has been suggested that this peak has a different physical origin and is related to a rapid suppression of the inelastic scattering rate in the superconducting state [43]. Such a drop of the inelastic scattering rate naturally appears when inelastic scattering in the normal state is dominated by electron-electron scattering, for example by spin fluctuations, because the superconducting gap is suppressing electron-electron scattering below the gap energy in the superconducting state. Detailed theoretical calculations by Hirschfeld et al [44] including $d$-wave superconductivity, strong impurity scattering, and inelastic spin fluctuation scattering were shown to be consistent with the experimental results.

In $\mathrm{MgB}_{2}$ microwave conductivity measurements have shown a conductivity peak appearing at about $0.5 T_{c}$ as shown in Fig. 9 This peak position seems to be somewhat intermediate between conventional superconductors and high- $T_{c}$ cuprates and the natural question arises, whether this is a coherence peak or a peak due to lifetime effects as in the cuprates. In order to address the question of lifetime effects a quick look at the temperature dependence of the resistivity in $\mathrm{MgB}_{2}$ is useful. It has been shown that the temperature dependence of the resistivity can be fitted well by Bloch-Grüneisen formula with a temperature independent residual resistivity associated with impurity scattering and a temperature dependent phonon scattering part [45]. This seems to show that no large electron-electron scattering is present in the normal state of $\mathrm{MgB}_{2}$. Moreover, at $T_{c}$ when superconductivity sets in, the resistivity is already in the saturation regime where phonon scattering is frozen out (due to the high frequency of the $\mathrm{E}_{2 g}$ 


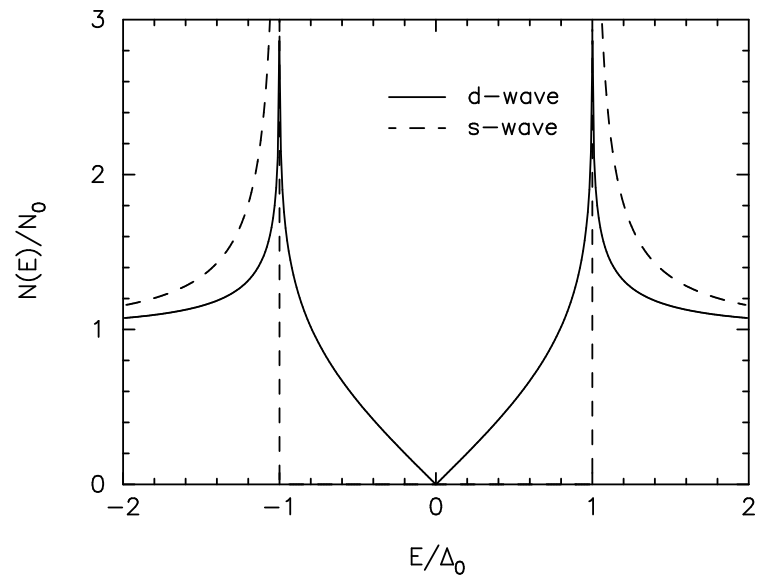

(a) Density of states

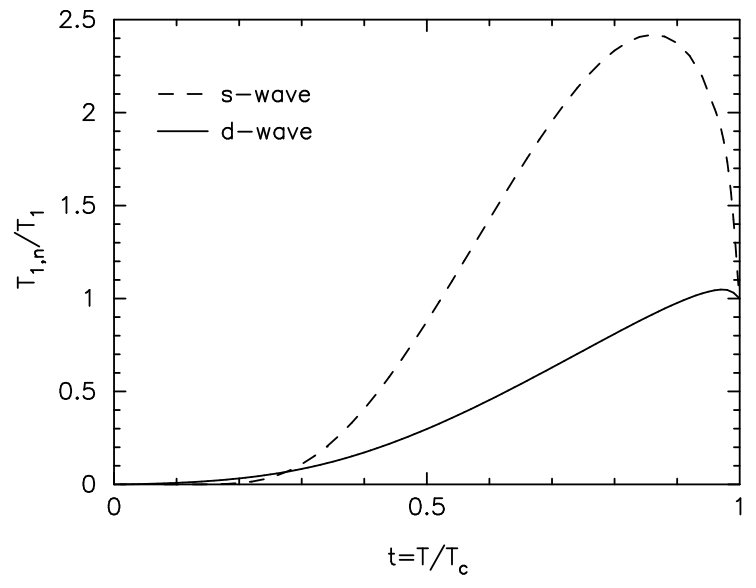

(b) NMR relaxation rate

Figure 8: (a) Density of states in a $d$-wave superconductor (solid line) and an $s$-wave superconductor (dashed line). (b) Corresponding NMR relaxation rates for $d$-wave (solid line) and $s$-wave (dashed line). In the $d$-wave case no large Hebel-Slichter peak is visible anymore.

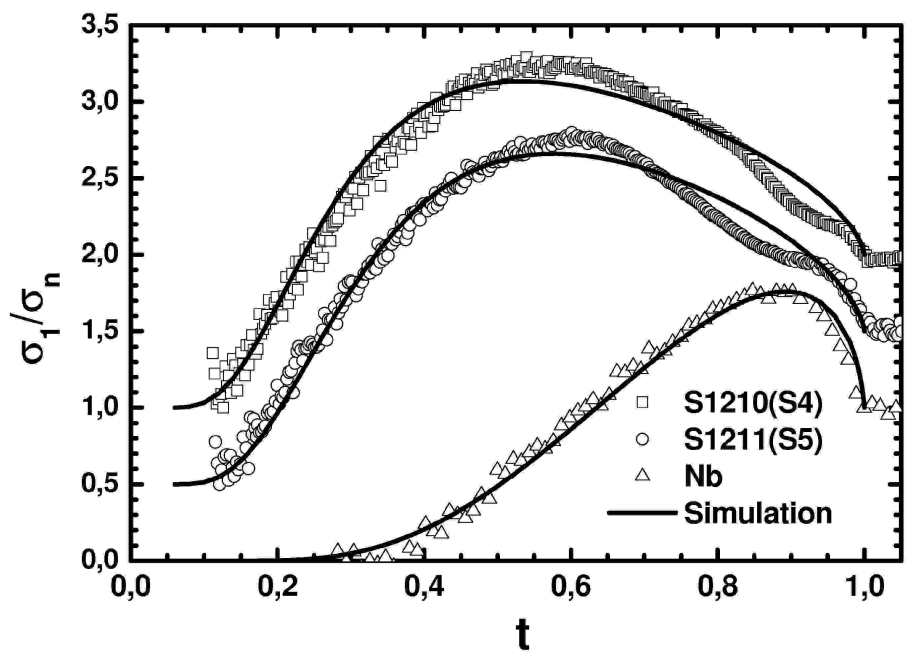

Figure 9: Temperature dependence of the microwave conductivity in $\mathrm{MgB}_{2}$ thin films (circles and squares). For comparison, the microwave conductivity of a Niobium thin film in the dirty limit is also shown (triangles). (Adapted from Ref. [33], (C)2003 The American Physical Society) 


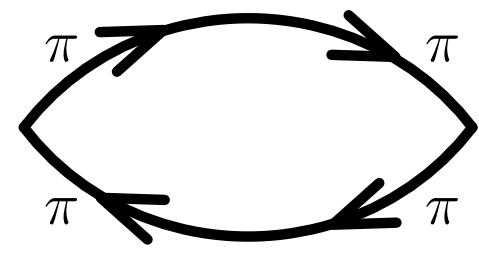

(a)

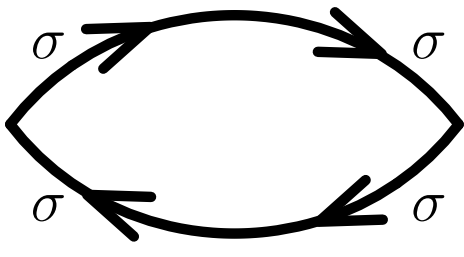

(b)

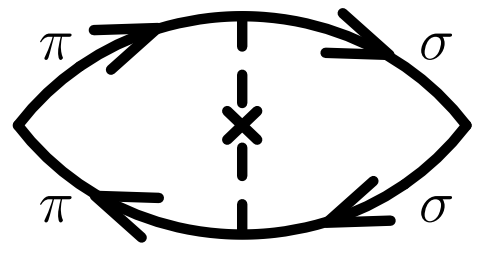

(c)

Figure 10: Diagrammatic contributions to the conductivity. (a) The main contribution to the $\pi$ band. (b) The main contribution to the $\sigma$ band. (c) The lowest order interband scattering contribution to the conductivity which is expected to be small in $\mathrm{MgB}_{2}$.

phonon mode). Therefore the resistivity at $T_{c}$ seems to be dominated by elastic impurity scattering and thus no rapid drop of inelastic scattering can be expected in the superconducting state. Note that this is in contrast to the high- $T_{c}$ cuprates, where resistivity varies linearly with temperature down to $T_{c}$ and is not saturated. These arguments seem to exclude the interpretation of the conductivity peak in $\mathrm{MgB}_{2}$ in terms of a lifetime effect.

In the following we therefore want to study the microwave conductivity of a dirty two gap superconductor. Dirty in the sense that the intraband impurity scattering rates in the two bands are assumed to be larger than the respective gaps, but interband scattering is neglected as suggested by the argument by Mazin et al. [16].

In a two band system like $\mathrm{MgB}_{2}$ we can approximate the total conductivity as the sum of the two partial conductivities of the two bands (parallel resistor formula). This approximation neglects interband scattering events like the one shown diagrammatically in Fig. 10 (c). However, these events are expected to give only small corrections, because interband impurity scattering is expected to be much weaker than intraband impurity scattering due to the argument by Mazin et al. [16].

If the intraband scattering rates in the two bands are sufficiently larger than their gaps, we can use the Mattis-Bardeen dirty limit formula for the conductivity in each band separately [46, 47]:

$$
\frac{\sigma_{\alpha}(\omega)}{\sigma_{n, \alpha}}=\frac{1}{2 \omega} \int_{-\infty}^{\infty} d \Omega\left(\tanh \frac{\Omega+\omega}{2 T}-\tanh \frac{\Omega}{2 T}\right)\left[N_{\alpha}(\Omega) N_{\alpha}(\Omega+\omega)+M_{\alpha}(\Omega) M_{\alpha}(\Omega+\omega)\right]
$$

Here, the normal and anomalous densities of states are given by

$$
N_{\alpha}(\Omega)=\operatorname{Re}\left\{\frac{|\Omega|}{\sqrt{\Omega^{2}-\Delta_{\alpha}^{2}}}\right\} \quad \text { and } \quad M_{\alpha}(\Omega)=\operatorname{Re}\left\{\frac{\Delta_{\alpha} \operatorname{sgn}(\Omega)}{\sqrt{\Omega^{2}-\Delta_{\alpha}^{2}}}\right\}
$$

The total conductivity under these assumptions is given by $\sigma_{1}(\omega)=\sigma_{\pi}(\omega)+\sigma_{\sigma}(\omega)$. The temperature dependence of the two gaps $\Delta_{\alpha}$ has to be determined from a solution of the two by 


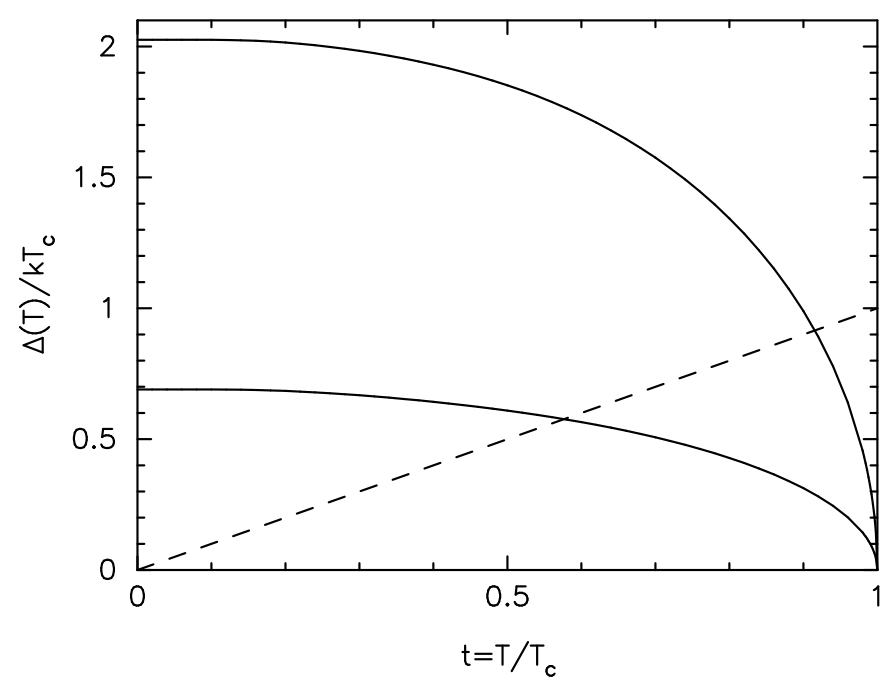

Figure 11: Temperature dependence of the two gaps in $\mathrm{MgB}_{2}$ found from a solution of the two by two gap equation. The dashed line shows $\Delta=k_{B} T$. Its crossing point with the small gap appears at a much lower temperature than the one with the large gap.

two gap equation:

$$
\Delta_{\alpha}=\sum_{\beta} \lambda^{\alpha \beta} \Delta_{\beta} \int_{0}^{\omega_{c}} d E \frac{\tanh \frac{\sqrt{E^{2}+\Delta_{\beta}^{2}}}{2 T}}{\sqrt{E^{2}+\Delta_{\beta}^{2}}}
$$

Here, $\omega_{c}$ is a characteristic phonon cut-off frequency and $\lambda^{\alpha \beta}$ the two by two coupling matrix. These parameters can be either taken from a band structure calculation or tried to be adjusted to the particular sample in question. In Ref. [33] an intermediate approach was taken: the partial densities of states and the cut-off frequency were taken from band structure calculations, while the remaining parameters were adjusted to the $T_{c}$ of the sample and the value of the small gap, which could be extracted from the exponential fall-off of the temperature dependent change of the penetration depth. A typical temperature dependence of the two gaps found this way is shown in Fig.11. One notices that the small gap reaches $k_{B} T$ at a much lower temperature than the large gap. According to the rule of thumb given above this would mean that the coherence peak in the $\pi$ band is expected to appear at much lower temperature than in a conventional superconductor.

That this is indeed the case is shown in Fig. 12. Here, the temperature dependence of the Mattis-Bardeen conductivity is shown for different zero temperature gap values. In these calculations the BCS temperature dependence has been scaled to different gap values for simplicity. Clearly, the position of the coherence peak follows the crossing point of the gap with $k_{B} T$.

In Fig. 9 the fits to the two $\mathrm{MgB}_{2}$ samples show the temperature dependence of the $\pi$ band contribution to the conductivity obtained using the temperature dependence of the small gap in Fig. 11. Apparently there is good agreement between measurement and calculation. The fit to the Niobium sample was obtained using the BCS temperature dependence of the gap, as one should expect for a conventional superconductor.

Now one might ask where the contribution from the $\sigma$ band in the $\mathrm{MgB}_{2}$ samples is. Accord- 

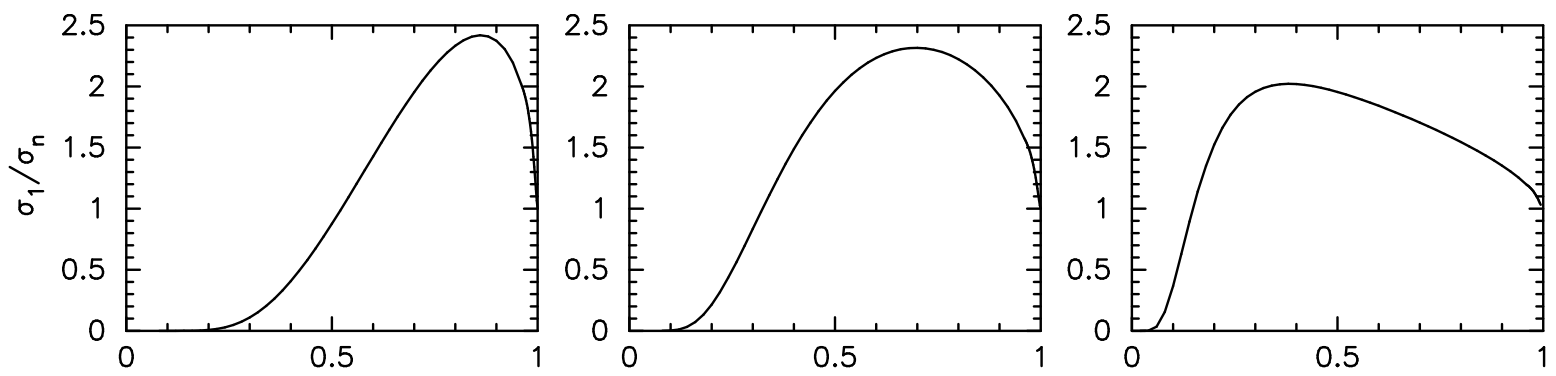

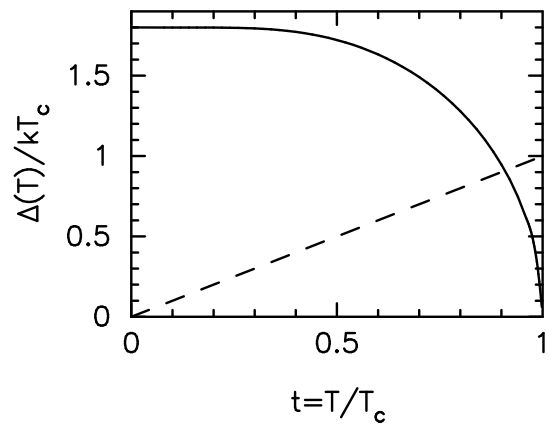

(a)

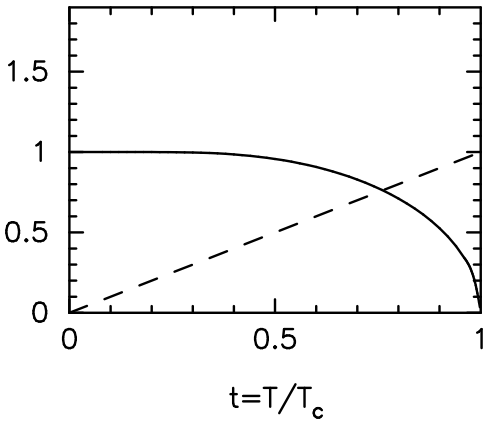

(b)

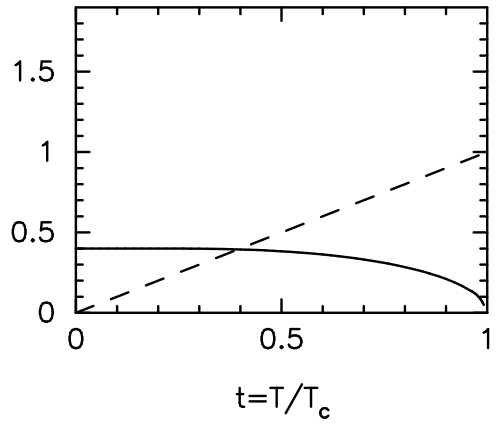

(c)

Figure 12: Temperature dependence of the microwave conductivity (upper panel) for different gap values (lower panel) at a frequency of $\omega=0.02 k_{B} T_{c}$.

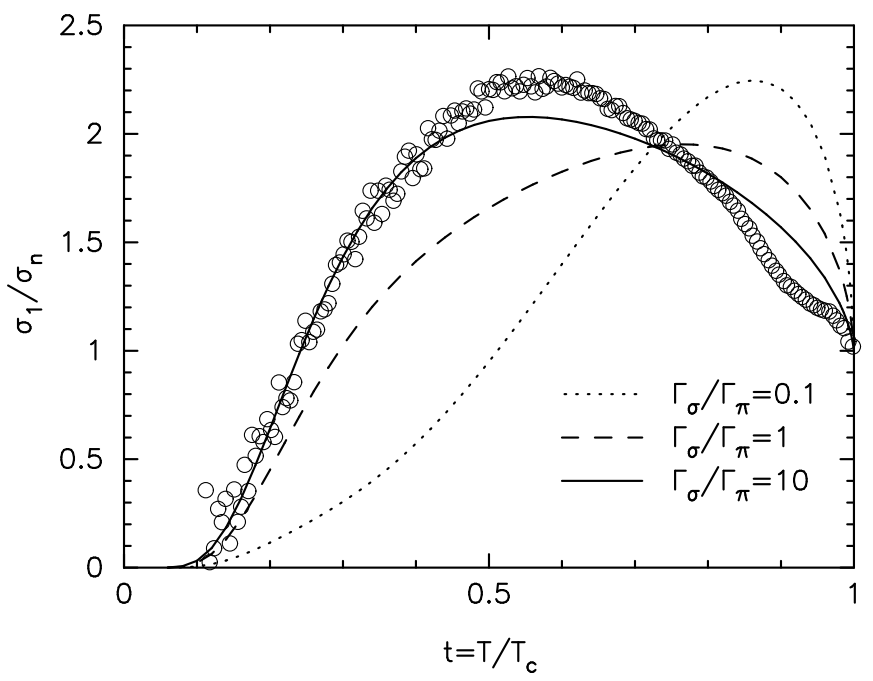

Figure 13: Temperature dependence of the microwave conductivity for different ratios of the scattering rates $\Gamma_{\sigma} / \Gamma_{\pi}=0.1,1$, and 10 along with the experimental results from Ref. [33]. 
ing to Fig. 12 the $\sigma$ band should produce a conventional coherence peak. The relative weight of the two contributions is determined by the partial normal state conductivities of the two bands, however. We have

$$
\sigma_{1}(\omega)=\sigma_{\pi}(\omega)+\sigma_{\sigma}(\omega)=\sigma_{n, \pi} \frac{\sigma_{\pi}(\omega)}{\sigma_{n, \pi}}+\sigma_{n, \sigma} \frac{\sigma_{\sigma}(\omega)}{\sigma_{n, \sigma}}
$$

where the conductivity ratios are given by Eq. (10). The normal state conductivities are related to the plasma frequencies $\omega_{p, \alpha}$ and the intraband scattering rates $\Gamma_{\alpha}$ via the expression

$$
\sigma_{n, \alpha}=\hbar \epsilon_{0} \frac{\omega_{p, \alpha}^{2}}{2 \Gamma_{\alpha}}
$$

Theoretical band structure values for the plasma frequencies have been given in Ref. [16]. According to these calculations we have $\omega_{p, \pi}=5.89 \mathrm{eV}$ and $\omega_{p, \sigma}=4.14 \mathrm{eV}$ for the in plane plasma frequencies. Therefore, if the scattering rates in the two bands are the same, the contribution of the $\pi$ band is expected to be a factor of 2 larger than the one of the $\sigma$ band. This is not sufficient to understand the apparent absence of a sizeable $\sigma$ band contribution in the experimental data. A possible explanation could be a stronger scattering rate in the $\sigma$ band at least in the thin film samples studied in Ref. [33]. To illustrate that, in Fig. 13 the temperature dependence of the conductivity is shown for different values of the relative ratio of the two scattering rates. These results suggest a at least 10 times larger scattering rate in the $\sigma$ band than in the $\pi$ band. In principle, the scattering rates in the two bands can be varied by selective doping, for example by doping with Aluminum for Magnesium or Carbon for Boron [48, 49, 16]. It would be interesting to see whether a cross-over like the one shown in Fig. 13 can be observed experimentally on a series of $\mathrm{MgB}_{2}$ films with varying degree of doping.

Concluding this section we have seen that the anomalous microwave conductivity peak observed in $\mathrm{MgB}_{2}$ thin films can be understood as a coherence peak due to the small gap. The peak appears at much lower temperatures than in conventional superconductors, because the small gap opens up more slowly upon reducing the temperature below $T_{c}$. As a result the condensation of excited quasiparticles and with it the exponential suppression of the conductivity sets in later. Again, this peculiar effect is consistent with two gap behavior and reinforces the picture presented in the previous sections.

\section{SUMMARY}

We have reviewed our present picture of superconductivity in magnesium diboride. Much of this picture stems from band structure calculations. We have seen that the high value of the critical temperature $T_{c}$ is due to a high frequency phonon mode that couples strongly to the electrons at the Fermi level. This coupling is particularly strong to the electrons on the $\sigma$ band and leads to a much larger superconducting gap on the $\sigma$ band than on the $\pi$ band. The different parity of the electronic wavefunctions of the $\sigma$ band and the $\pi$ band results in a strong reduction of the interband impurity scattering matrix element. This makes the two different gaps particularly stable against impurity scattering. For these reasons magnesium diboride appears to be the clearest example of an intrinsic two gap superconductor to date.

Two gap superconductivity in magnesium diboride leads to unusual behavior in a number of experimentally accessible quantities. Here, we have focused on the temperature dependence 
of the upper critical field anisotropy and the microwave conductivity. The upper critical field anisotropy shows an unusual strong temperature dependence. This feature is related to the presence of the two gaps, but also to the very different anisotropy of the two bands. Here, the intermediate strength of the interband pairing interaction plays a crucial role as well. The temperature dependence of the microwave conductivity shows a peak at temperatures around $0.5 T_{c}$. This peak can be understood as an anomalous coherence peak that is shifted downwards in temperatures because of the small gap.

In conclusion, the case of two gap superconductivity in magnesium diboride generated by strong electron-phonon coupling appears to be well established and can be consistently observed in very different experimental quantities such as the upper critical field or the microwave conductivity.

The author would like to thank O. V. Dolgov, S. Graser, C. Iniotakis, B. B. Jin, N. Klein, S.-I. Lee, K. Maki, A. I. Posazhennikova, and N. Schopohl for valuable discussions about this and related topics. 


\section{APPENDIX}

In this appendix the variational method for the calculation of the upper critical field from Eqs. (5) and (6) shall be described. This method was used in Refs. [23, 30].

Defining the operator

$$
L_{\alpha}=2\left|\omega_{n}\right|+\operatorname{sgn} \omega_{n} \vec{v}_{F, \alpha}(\hat{k})\left[\hbar \vec{\nabla}-i \frac{2 e}{c} \vec{A}(\vec{r})\right]
$$

where $\vec{v}_{F, \alpha}(\hat{k})$ is the Fermi velocity of band $\alpha, \vec{A}$ the vector potential due to the internal magnetic field within the system, and $\omega_{n}=(2 n+1) \pi T$ the Matsubara frequencies, Eq. (5) can be inverted using the identity

$$
L_{\alpha}^{-1}=\int_{0}^{\infty} d s e^{-s L_{\alpha}}
$$

which leads to

$$
f_{\alpha}\left(\vec{r}, \hat{k} ; \omega_{n}\right)=-2 \int_{0}^{\infty} d s e^{-s L_{\alpha}} \Delta_{\alpha}(\vec{r})
$$

Introducing this into the gap equation Eq. (6) we can eliminate $f_{\alpha}$ :

$$
\Delta_{\alpha}(\vec{r})=2 \pi T \sum_{\alpha^{\prime}} \sum_{\left|\omega_{n}\right|<\omega_{c}} \lambda^{\alpha \alpha^{\prime}}\left\langle\int_{0}^{\infty} d s e^{-s L_{\alpha^{\prime}}} \Delta_{\alpha^{\prime}}(\vec{r})\right\rangle_{\alpha^{\prime}}
$$

This equation is a linear equation for $\Delta_{\alpha}(\vec{r})$.

As is usual in weak-coupling theory we can eliminate the cutoff frequency $\omega_{c}$ in favor of the critical temperature $T_{c}$. For this purpose we consider Eq. (18) in the absence of a magnetic field at $T_{c}$. Then the gap function $\Delta_{\alpha}(\vec{r})$ becomes homogeneous and we find

$$
\begin{aligned}
\Delta_{\alpha} & =2 \pi T_{c} \sum_{\left|\omega_{n}\left(T_{c}\right)\right|<\omega_{c}} \int_{0}^{\infty} d s e^{-2 s\left|\omega_{n}\left(T_{c}\right)\right|} \sum_{\alpha^{\prime}} \lambda^{\alpha \alpha^{\prime}} \Delta_{\alpha^{\prime}} \\
& =2 \pi T_{c} \sum_{\omega_{n}\left(T_{c}\right)>0}^{\omega_{c}} \frac{1}{\omega_{n}\left(T_{c}\right)} \sum_{\alpha^{\prime}} \lambda^{\alpha \alpha^{\prime}} \Delta_{\alpha^{\prime}}
\end{aligned}
$$

This is an eigenvalue equation for $\Delta_{\alpha}$ and the largest eigenvalue $\lambda_{+}$of the matrix $\lambda^{\alpha \alpha^{\prime}}$ determines $T_{c}$. Thus we find

$$
\frac{1}{\lambda_{+}}=2 \pi T_{c} \sum_{\omega_{n}\left(T_{c}\right)>0}^{\omega_{c}} \frac{1}{\omega_{n}\left(T_{c}\right)}
$$

For $\omega_{c} \gg T_{c}$ the following relation holds:

$$
2 \pi T_{c} \sum_{\omega_{n}\left(T_{c}\right)>0}^{\omega_{c}} \frac{1}{\omega_{n}\left(T_{c}\right)}-2 \pi T \sum_{\omega_{n}(T)>0}^{\omega_{c}} \frac{1}{\omega_{n}(T)} \simeq \ln \frac{T}{T_{c}}
$$

and thus we can write Eq. (20) in the form

$$
\frac{1}{\lambda_{+}}-\ln \frac{T}{T_{c}}=2 \pi T \sum_{\omega_{n}(T)>0}^{\omega_{c}} \frac{1}{\omega_{n}(T)}=2 \pi T \sum_{\left|\omega_{n}(T)\right|<\omega_{c}} \int_{0}^{\infty} d s e^{-2 s\left|\omega_{n}(T)\right|}
$$


Multiplying this equation by $\sum_{\alpha^{\prime}} \lambda^{\alpha \alpha^{\prime}} \Delta_{\alpha^{\prime}}(\vec{r})$ it can be subtracted from Eq. (18) leading to

$$
\begin{aligned}
& \Delta_{\alpha}(\vec{r})+\sum_{\alpha^{\prime}} \lambda^{\alpha \alpha^{\prime}} \Delta_{\alpha^{\prime}}(\vec{r})\left(-\frac{1}{\lambda_{+}}+\ln \frac{T}{T_{c}}\right) \\
& =\sum_{\alpha^{\prime}} \lambda^{\alpha \alpha^{\prime}} 2 \pi T \sum_{\left|\omega_{n}\right|<\omega_{c}}\left\langle\int_{0}^{\infty} d s\left[e^{-s L_{\alpha^{\prime}}}-e^{-2 s\left|\omega_{n}\right|}\right] \Delta_{\alpha^{\prime}}(\vec{r})\right\rangle_{\alpha^{\prime}} \\
& =\sum_{\alpha^{\prime}} \lambda^{\alpha \alpha^{\prime}} 4 \pi T \sum_{\omega_{n}>0}^{\omega_{c}} \int_{0}^{\infty} d s e^{-2 s \omega_{n}}\left\langle\left[e^{-i s \vec{v}_{F, \alpha^{\prime}} \cdot \vec{\Pi}}-1\right] \Delta_{\alpha^{\prime}}(\vec{r})\right\rangle_{\alpha^{\prime}}
\end{aligned}
$$

Here, we have eliminated the sgn $\omega_{n}$ factor assuming inversion symmetry of the Fermi velocity $\vec{v}_{F, \alpha}(\hat{k})=-\vec{v}_{F, \alpha}(-\hat{k})$ and introduced the operator

$$
\vec{\Pi}=\frac{\hbar}{i} \vec{\nabla}-\frac{2 e}{c} \vec{A}(\vec{r})
$$

In Eq. 23 we may now extend the $\omega_{n}$ summation to infinity and sum it up:

$$
\begin{aligned}
\sum_{\omega_{n}>0} e^{-2 s \omega_{n}} & =\sum_{n=0}^{\infty} e^{-2 s(2 n+1) \pi T}=e^{-2 s \pi T} \sum_{n=0}^{\infty}\left(e^{-4 s \pi T}\right)^{n} \\
& =e^{-2 s \pi T} \frac{1}{1-e^{-4 s \pi T}}=\frac{1}{2 \sinh (2 s \pi T)}
\end{aligned}
$$

Note that integration and summation in Eq. (23) may only be interchanged because the divergence for $s \rightarrow 0$ has been eliminated. (In Eq. (18) this summation was not possible). So we find from Eq. (23):

$$
\begin{aligned}
& \Delta_{\alpha}(\vec{r})+\sum_{\alpha^{\prime}} \lambda^{\alpha \alpha^{\prime}} \Delta_{\alpha^{\prime}}(\vec{r})\left(-\frac{1}{\lambda_{+}}+\ln \frac{T}{T_{c}}\right) \\
& \quad=\sum_{\alpha^{\prime}} \lambda^{\alpha \alpha^{\prime}} \int_{0}^{\infty} \frac{d u}{\sinh u}\left\langle\left[e^{-i u \vec{v}_{F, \alpha^{\prime}} \cdot \vec{\Pi} /(2 \pi T)}-1\right] \Delta_{\alpha^{\prime}}(\vec{r})\right\rangle_{\alpha^{\prime}}
\end{aligned}
$$

In the presence of an external magnetic field $\vec{B}=\vec{\nabla} \times \vec{A}$ it is convenient to choose the field direction as the $z$-axis of the coordinate system. In these coordinates $\Delta_{\alpha}(\vec{r})$ does not depend on $z$. Choosing the gauge $\vec{A}=B x \hat{y}$ the operator $\vec{\Pi}$ simplifies to

$$
\vec{\Pi}=\frac{\hbar}{i} \vec{\nabla}-\frac{2 e}{c} \vec{A}(\vec{r})=\left(\begin{array}{c}
-i \hbar \partial_{x} \\
-i \hbar \partial_{y}-\frac{2 e}{c} B x \\
0
\end{array}\right)=\sqrt{\frac{e B}{c}}\left(\begin{array}{c}
a+a^{\dagger} \\
i\left(a-a^{\dagger}\right) \\
0
\end{array}\right)
$$

where we have introduced raising and lowering operators $2 \sqrt{\frac{e B}{c}} a=-i \hbar \partial_{x}-\hbar \partial_{y}+2 i \frac{e B}{c} x$ and $2 \sqrt{\frac{e B}{c}} a^{\dagger}=-i \hbar \partial_{x}+\hbar \partial_{y}-2 i \frac{e B}{c} x$. Thus, we see from Eq. (26) that only the components of the Fermi velocity perpendicular to the magnetic field direction play a role and we have

$$
\vec{v}_{F, \alpha} \cdot \vec{\Pi}=\sqrt{\frac{e B}{c}}\left[\left(v_{x, \alpha}+i v_{y, \alpha}\right) a+\left(v_{x, \alpha}-i v_{y, \alpha}\right) a^{\dagger}\right]
$$


where $v_{x, \alpha}$ and $v_{y, \alpha}$ are the components of the Fermi velocity perpendicular to the magnetic field $\vec{B}$. Note, that these components are not necessarily identical to the components of the Fermi velocity with respect to the crystal axes in Eq. (4).

It is useful to introduce a scaling of the $x$ - and $y$-coordinates of the form

$$
x=e^{\tau} \bar{x} \quad y=e^{-\tau} \bar{y}
$$

Here $e^{\tau}$ is a scaling factor which scales the $x$ - and $y$-coordinates in such a way as to preserve the area. Expressed in the new coordinates $\bar{x}$ and $\bar{y}$ the operator $\vec{\Pi}$ can be written:

$$
\vec{\Pi}=\left(\begin{array}{c}
-i \hbar e^{-\tau} \partial_{\bar{x}} \\
-i \hbar e^{\tau} \partial_{\bar{y}}-\frac{2 e}{c} e^{\tau} B \bar{x} \\
0
\end{array}\right)=\sqrt{\frac{e B}{c}}\left(\begin{array}{c}
e^{-\tau}\left(\bar{a}+\bar{a}^{\dagger}\right) \\
i e^{\tau}\left(\bar{a}-\bar{a}^{\dagger}\right) \\
0
\end{array}\right)
$$

where the raising and lowering operators $\bar{a}$ and $\bar{a}^{\dagger}$ are also expressed in terms of these new coordinates. Using this result we find

$$
\vec{v}_{F, \alpha} \cdot \vec{\Pi}=\sqrt{\frac{e B}{c}}\left[\left(e^{-\tau} v_{x, \alpha}+i e^{\tau} v_{y, \alpha}\right) \bar{a}+\left(e^{-\tau} v_{x, \alpha}-i e^{\tau} v_{y, \alpha}\right) \bar{a}^{\dagger}\right]
$$

We can now write the exponential operator in Eq. (26) as follows:

$$
e^{-i u \vec{v}_{F, \alpha} \cdot \vec{\Pi} /(2 \pi T)}=e^{A+B}
$$

with

$$
\begin{aligned}
& A=-i \frac{u}{2 \pi T} \sqrt{\frac{e B}{c}}\left(e^{-\tau} v_{x, \alpha}-i e^{\tau} v_{y, \alpha}\right) \bar{a}^{\dagger} \\
& B=-i \frac{u}{2 \pi T} \sqrt{\frac{e B}{c}}\left(e^{-\tau} v_{x, \alpha}+i e^{\tau} v_{y, \alpha}\right) \bar{a}
\end{aligned}
$$

Using the identity

$$
e^{A+B}=e^{-[A, B] / 2} e^{A} e^{B}
$$

and calculating the commutator

$$
[A, B]=u^{2} \frac{e B}{c(2 \pi T)^{2}}\left(e^{-2 \tau} v_{x, \alpha}^{2}+e^{2 \tau} v_{y, \alpha}^{2}\right)
$$

we can write

$$
\begin{aligned}
& e^{-i u \vec{v}_{F, \alpha} \cdot \vec{\Pi} /(2 \pi T)}= \\
& \quad e^{-u^{2} \frac{e B}{2 c(2 \pi T)^{2}}\left(e^{-2 \tau} v_{x, \alpha}^{2}+e^{2 \tau} v_{y, \alpha}^{2}\right)} e^{-i \frac{u}{2 \pi T} \sqrt{\frac{e B}{c}}\left(e^{-\tau} v_{x, \alpha}-i e^{\tau} v_{y, \alpha}\right) \bar{a}^{\dagger}} e^{-i \frac{u}{2 \pi T} \sqrt{\frac{e B}{c}}\left(e^{-\tau} v_{x, \alpha}+i e^{\tau} v_{y, \alpha}\right) \bar{a}}
\end{aligned}
$$

In principle, we can view Eq. (26) as an eigenvalue problem. The highest eigenvalue and its corresponding eigenfunction of the right hand side operator will determine the upper critical field $B_{c 2}(T)$. We already know from Abrikosov's solution of Ginzburg-Landau theory that the 
solution for an isotropic $s$-wave superconductor will be Abrikosov's vortex state wave function $\psi_{\Lambda}(\vec{r})$. This wavefunction has the property that it is destroyed by the operator $a$

$$
a \psi_{\Lambda}(\vec{r})=0
$$

and has the form of the lowest Landau level wavefunction. In principle, one can try to solve Eq. (26) by making a Landau level expansion of $\Delta_{\alpha}(\vec{r})$ [32, 50]. However, for an anisotropic superconductor one should expect a distortion of the vortex lattice. Therefore here we will start from a variational ansatz to Eq. (26) by choosing a different wavefunction, which obeys the equation

$$
\bar{a} \Delta_{\alpha}(\vec{r})=0
$$

This corresponds to the choice Eq. 8 . 8 . Here, we can use the scaling parameter $\tau$ now as a variational parameter which has to be adjusted such as to yield the highest possible eigenvalue of Eq. (26). If we insert this variational wavefunction into Eq. (26) the $e^{B}$ and $e^{A}$ operators just drop out and we are left with the equation

$$
\Delta_{\alpha}=\sum_{\alpha^{\prime}} \lambda^{\alpha \alpha^{\prime}}\left[\frac{1}{\lambda_{+}}-\ln \frac{T}{T_{c}}-l_{\alpha^{\prime}}\left(\tau, \frac{B_{c 2}}{T^{2}}\right)\right] \Delta_{\alpha^{\prime}}
$$

where the function $l_{\alpha}$ is given by the expression

$$
l_{\alpha}\left(\tau, \frac{B_{c 2}}{T^{2}}\right)=\int_{0}^{\infty} \frac{d u}{\sinh u}\left\langle 1-e^{-u^{2} \frac{e B_{c 2}}{8 c \pi^{2} T^{2}}\left(e^{-2 \tau} v_{x, \alpha}^{2}(\hat{k})+e^{2 \tau} v_{y, \alpha}^{2}(\hat{k})\right)}\right\rangle_{\alpha}
$$

Equation (38) is a $2 \times 2$ matrix equation and the upper critical field is determined by the criterion that the largest eigenvalue of the right hand side becomes 1 . This criterion leads to the characteristic equation

$$
(1-\eta) l_{\sigma}+\eta l_{\pi}+\ln t=-\Lambda_{ \pm}\left(l_{\sigma}+\ln t\right)\left(l_{\pi}+\ln t\right)
$$

where $t=T / T_{c}$ and the parameters $\eta$ and $\Lambda_{ \pm}$are given by

$$
\eta=\frac{\lambda^{\pi \pi}-\lambda_{-}}{\lambda_{+}-\lambda_{-}} \quad \text { and } \quad \Lambda_{ \pm}=\frac{\lambda_{+} \lambda_{-}}{\lambda_{+}-\lambda_{-}}
$$

Here, $\lambda_{+}$and $\lambda_{-}$are the larger and smaller eigenvalue of the matrix $\lambda^{\alpha \alpha^{\prime}}$, respectively. Note, that Eq. (40) means that only the two parameters $\eta$ and $\Lambda_{ \pm}$out of the four parameters $\lambda^{\alpha \alpha^{\prime}}$ determine the upper critical field of a two gap superconductor.

Equation (40) is a quadratic equation in $\ln t$ and allows to calculate $t$ once $l_{\pi}$ and $l_{\sigma}$ are known. Therefore, calculation of $B_{c 2}$ from Eqs. (39) and (40) can proceed as follows: for given values of $B_{c 2} / T^{2}$ and $\tau$ the quantities $l_{\pi}$ and $l_{\sigma}$ are calculated using Eq. (39) and the Fermi velocities given in Eq. (4). Then $t$ can be calculated using Eq. (40) and the matrix elements given in Eq. (7). Now, the parameter $\tau$ is optimized such as to maximize $t$. From the maximized $t$ and the given value of $B_{c 2} / T^{2}$ finally $B_{c 2}$ can be calculated. This procedure is repeated for several values of $B_{c 2} / T^{2}$ producing a $B_{c 2}(T)$ curve. 
In Eq. (39) the integration over $u$ has a special form and it is useful to introduce the integral

$$
I(y)=\int_{0}^{\infty} \frac{d u}{\sinh u}\left(1-e^{-y u^{2}}\right)
$$

While this integral cannot be calculated analytically in the most general case, it at least possesses some simple limiting expressions for small and large arguments $y$. (Large $y$ corresponds to the zero temperature limit in Eq. (39), while small $y$ corresponds to $T \rightarrow T_{c}$, when $B_{c 2} \rightarrow 0$.) These limiting expressions read

$$
I(y)=\left\{\begin{array}{cl}
\frac{7}{2} \zeta(3) y-\frac{93}{4} \zeta(5) y^{2} & \text { for } \quad y \ll 1 \\
\frac{1}{2} \ln (4 \gamma y) & \text { for } \quad y \gg 1
\end{array}\right.
$$

Here, $\zeta(n)$ is the Riemann Zeta function and $\ln \gamma=0.577215$ Euler's constant. Using the integral $I(y)$ Eq. (39) can be written

$$
l_{\alpha}\left(\tau, \frac{B_{c 2}}{T^{2}}\right)=\left\langle I\left[\frac{e B_{c 2}}{8 c \pi^{2} T^{2}}\left(e^{-2 \tau} v_{x, \alpha}^{2}(\hat{k})+e^{2 \tau} v_{y, \alpha}^{2}(\hat{k})\right)\right]\right\rangle_{\alpha}
$$

Using Eq. (43) this allows to find simple limiting expressions for $l_{\alpha}$ in the limits $T \rightarrow 0$ and $T \rightarrow T_{c}$. In particular, in the limit $T \rightarrow T_{c}$ we have in linear order in $B_{c 2}$

$$
l_{\alpha}=\frac{7}{2} \zeta(3) \frac{e B_{c 2}}{8 c \pi^{2} T_{c}^{2}}\left(e^{-2 \tau}\left\langle v_{x, \alpha}^{2}\right\rangle_{\alpha}+e^{2 \tau}\left\langle v_{y, \alpha}^{2}\right\rangle_{\alpha}\right)
$$

Since $B_{c 2}$ goes to zero for $T \rightarrow T_{c}$, both $l_{\alpha}$ and $\ln t$ decrease linearly and the right hand side of Eq. (40) can be neglected, because it becomes quadratic in $1-t$. Thus in linear order we have

$$
\begin{aligned}
-\ln & \frac{T}{T_{c}}=(1-\eta) l_{\sigma}+\eta l_{\pi}=\frac{7 \zeta(3) e B_{c 2}}{16 c \pi^{2} T_{c}^{2}} \\
& \cdot\left\{e^{-2 \tau}\left[(1-\eta)\left\langle v_{x, \sigma}^{2}\right\rangle_{\sigma}+\eta\left\langle v_{x, \pi}^{2}\right\rangle_{\pi}\right]+e^{2 \tau}\left[(1-\eta)\left\langle v_{y, \sigma}^{2}\right\rangle_{\sigma}+\eta\left\langle v_{y, \pi}^{2}\right\rangle_{\pi}\right]\right\}
\end{aligned}
$$

Minimizing this expression with respect to $\tau$ one finds

$$
e^{2 \tau}=\sqrt{\frac{(1-\eta)\left\langle v_{x, \sigma}^{2}\right\rangle_{\sigma}+\eta\left\langle v_{x, \pi}^{2}\right\rangle_{\pi}}{(1-\eta)\left\langle v_{y, \sigma}^{2}\right\rangle_{\sigma}+\eta\left\langle v_{y, \pi}^{2}\right\rangle_{\pi}}}
$$

Using this result we can calculate the slope of $B_{c 2}$ at $T_{c}$ from Eq. (46):

$$
\left.\frac{d B_{c 2}}{d T}\right|_{T_{c}}=\frac{8 c \pi^{2} T_{c}}{7 \zeta(3) e} \frac{1}{\sqrt{\left[(1-\eta)\left\langle v_{x, \sigma}^{2}\right\rangle_{\sigma}+\eta\left\langle v_{x, \pi}^{2}\right\rangle_{\pi}\right]\left[(1-\eta)\left\langle v_{y, \sigma}^{2}\right\rangle_{\sigma}+\eta\left\langle v_{y, \pi}^{2}\right\rangle_{\pi}\right]}}
$$




\section{REFERENCES}

[1] J. Nagamatsu, N. Nakagawa, T. Muranaka, Y. Zenitani, and J. Akimitsu, Nature 410 (2001) 63.

[2] P. C. Canfield and G. W. Crabtree, Physics Today 56, No. 3 (2003) 34.

[3] S. L. Bud'ko, G. Lapertot, C. Petrovic, C. E. Cunningham, N. Anderson, and P. C. Canfield, Phys. Rev. Lett. 86 (2001) 1877.

[4] F. Bouquet, R. A. Fisher, N. E. Phillips, D. G. Hinks, and J. D. Jorgensen, Phys. Rev. Lett. 87 (2001) 047001.

[5] R. Prozorov, R. W. Giannetta, S. L. Bud'ko, and P. C. Canfield, Phys. Rev. B 64 (2001) 180501(R).

[6] J. Kortus, I. I. Mazin, K. D. Belashchenko, V. P. Antropov, and L. L. Boyer, Phys. Rev. Lett. 86 (2001) 4656.

[7] Y. Kong, O. V. Dolgov, O. Jepsen, and O. K. Andersen, Phys. Rev. B 64 (2001) 020501(R).

[8] K.-P. Bohnen, R. Heid, and B. Renker, Phys. Rev. Lett. 86 (2001) 5771.

[9] A. Y. Liu, I. I. Mazin, and J. Kortus, Phys. Rev. Lett. 87 (2001) 087005.

[10] For an early review see Fig. 35 in C. Buzea and Yamashita, Superconductor Science and Techn. 14 (2001) R115.

[11] M. R. Eskildsen, M. Kugler, S. Tanaka, J. Jun, S. M. Kazakov, J. Karpinski, and Ø. Fischer, Phys. Rev. Lett. 89 (2002) 187003.

[12] A. A. Golubov, J. Kortus, O. V. Dolgov, O. Jepsen, Y. Kong, O. K. Andersen, B. J. Gibson, K. Ahn, and R. K. Kremer, J. Phys.: Condens. Matter 14 (2002), 1353.

[13] H. J. Choi, D. Roundy, H. Sun, M. L. Cohen, and S. G. Louie, Phys. Rev. B 66 (2002) 020513(R).

[14] H. J. Choi, D. Roundy, H. Sun, M. L. Cohen, and S. G. Louie, Nature (London) 418 (2002) 758.

[15] N. Schopohl and K. Scharnberg, Solid State Comm. 22 (1977) 371; N. Schopohl, Diploma Thesis, University of Hamburg (1977).

[16] I. I. Mazin, O. K. Andersen, O. Jepsen, O. V. Dolgov, J. Kortus, A. A. Golubov, A. B. Kuz'menko, and D. van der Marel, Phys. Rev. Lett. 89 (2002) 107002.

[17] O. V. Dolgov, R. S. Gonnelli, G. A. Ummarino, A. A. Golubov, S. V. Shulga, and J. Kortus, Phys. Rev. B 68 (2003) 132503.

[18] M. Angst, R. Puzniak, A. Wisniewski, J. Jun, S. M. Kazakov, J. Karpinski, J. Roos, and H. Keller, Phys. Rev. Lett. 88 (2002) 167004.

[19] Yu. Eltsev, S. Lee, K. Nakao, N. Chikumoto, S. Tajima, N. Koshizuka, and M. Murakami, Phys. Rev. B 65 (2002) 140501(R).

[20] L. Lyard, P. Samuely, P. Szabo, T. Klein, C. Marcenat, L. Paulius, K. H. P. Kim, C. U. Jung, H.-S. Lee, B. Kang, S. Choi, S.-I. Lee, J. Marcus, S. Blanchard, A. G. M. Jansen, U. Welp, G. Karapetrov, and W. K. Kwok, Phys. Rev. B 66 (2002) 180502(R).

[21] U. Welp, A. Rydh, G. Karapetrov, W. K. Kwok, and G. W. Crabtree, Ch. Marcenat, L. Paulius, T. Klein, J. Marcus, K. H. P. Kim, C. U. Jung, H.-S. Lee, B. Kang, and S.-I. Lee, Phys. Rev. B 67 (2003) 012505.

[22] S. V. Shulga, S.-L. Drechsler, G. Fuchs, K.-H. Müller, K. Winzer, M. Heinecke, and K. Krug, Phys. Rev. Lett. 80 (1998) 1730. 
[23] A. I. Posazhennikova, T. Dahm, and K. Maki, Europhys. Lett. 60 (2002) 134.

[24] B. B. Jin, N. Klein, W. N. Kang, Hyeong-Jin Kim, Eun-Mi Choi, Sung-Ik Lee, T. Dahm, and K. Maki, Phys. Rev. B 66 (2002) 104521.

[25] T. Dahm, A. I. Posazhennikova, and K. Maki, Acta Phys. Pol. B 34 (2003) 549.

[26] L. Lyard, P. Szabo, T. Klein, J. Marcus, C. Marcenat, K. H. Kim, B. W. Kang, H. S. Lee, and S. I. Lee, Phys. Rev. Lett. 92 (2004) 057001.

[27] R. Cubitt, M. R. Eskildsen, C. D. Dewhurst, J. Jun, S. M. Kazakov, and J. Karpinski, Phys. Rev. Lett. 91 (2003) 047002.

[28] M. Xu, H. Kitazawa, Y. Takano, J. Ye, K. Nishida, H. Abe, A. Matsushita, N. Tsujii, and G. Kido, Appl. Phys. Lett. 79 (2001) 2779.

[29] P. Miranovic, K. Machida, and V. G. Kogan, J. Phys. Soc. Japan 72 (2003) 221.

[30] T. Dahm and N. Schopohl, Phys. Rev. Lett. 91 (2003) 017001.

[31] S. Graser, T. Dahm, and N. Schopohl, Phys. Rev. B 69 (2004) 014511.

[32] C. T. Rieck, K. Scharnberg, and N. Schopohl, J. Low Temp. Phys. 84 (1991) 381.

[33] B. B. Jin, T. Dahm, A. I. Gubin, Eun-Mi Choi, Hyun Jung Kim, Sung-IK Lee, W. N. Kang, and N. Klein, Phys. Rev. Lett. 91 (2003) 127006.

[34] A. Rydh, U. Welp, A. E. Koshelev, W. K. Kwok, G. W. Crabtree, R. Brusetti, L. Lyard, T. Klein, C. Marcenat, B. Kang, K. H. Kim, K. H. P. Kim, H.-S. Lee, S.-I. Lee, cond-mat/0308319.

[35] T. Dahm, S. Graser, and N. Schopohl, Physica C 408-410 (2004) 336 (cond-mat/0304194).

[36] G. Eilenberger, Z. Phys. 214 (1968) 195.

[37] A. I. Larkin and Yu. N. Ovchinnikov, Zh. Eksp. Teor. Fiz. 55 (1968) 2262 - engl. transl. Sov. Phys. JETP 28 (1969) 1200.

[38] A. Gurevich, Phys. Rev. B 67 (2003) 184515.

[39] A. A. Golubov and A. E. Koshelev, Phys. Rev. B 68 (2003) 104503.

[40] A. E. Koshelev and A. A. Golubov, Phys. Rev. Lett. 92 (2004) 107008.

[41] F. Marsiglio, Phys. Rev. B 44 (1991) 5373.

[42] A. Hosseini, R. Harris, S. Kamal, P. Dosanjh, J. Preston, R. Liang, W. N. Hardy, and D. A. Bonn, Phys. Rev. B 60 (1999) 1349.

[43] D. A. Bonn, P. Dosanjh, R. Liang, and W. N. Hardy, Phys. Rev. Lett. 68 (1992) 2390.

[44] P. J. Hirschfeld, W. O. Putikka, and D. J. Scalapino, Phys. Rev. B 50 (1994) 10250.

[45] Kijoon H. P. Kim, Jae-Hyuk Choi, C. U. Jung, P. Chowdhury, Hyun-Sook Lee, Min-Seok Park, Heon-Jung Kim, J. Y. Kim, Zhonglian Du, Eun-Mi Choi, Mun-Seog Kim, W. N. Kang, Sung-Ik Lee, G. Y. Sung, and J. Y. Lee, Phys. Rev. B 65 (2002) 100510(R).

[46] D. C. Mattis and J. Bardeen, Phys. Rev. 111 (1958) 412.

[47] S. B. Nam, Phys. Rev. 156 (1967) 470.

[48] V. Braccini, A. Gurevich, J.E. Giencke, M.C. Jewell, C.B. Eom, D.C. Larbalestier, A. Pogrebnyakov, Y. Cui, B. T. Liu, Y. F.Hu, J. M. Redwing, Qi Li, X.X. Xi, R.K. Singh, R. Gandikota, J. Kim, B. Wilkens, N. Newman, J. Rowell, B. Moeckly, V. Ferrando, C. Tarantini, D. Marre, M. Putti, C. Ferdeghini, R. Vaglio, E. Haanappel, cond-mat/0402001

[49] M.Putti, C.Ferdeghini, M.Monni, I.Pallecchi, C.Tarantini, P.Manfrinetti, A.Palenzona, D.Daghero, R.S.Gonnelli, V.A. Stepanov, cond-mat/0406377.

[50] Ye Sun and K. Maki, Phys. Rev. B 47, 9108 (1993); H. Won and K. Maki, Phys. Rev. B 53, 5927 (1996). 